NBER WORKING PAPER SERIES

\title{
INFORMATION DYNAMICS AND EQUILIBRIUM MULTIPLICITY IN GLOBAL GAMES OF REGIME CHANGE
}

\author{
George-Marios Angeletos \\ Christian Hellwig \\ Alessandro Pavan \\ Working Paper 11017 \\ http://www.nber.org/papers/w11017 \\ NATIONAL BUREAU OF ECONOMIC RESEARCH \\ 1050 Massachusetts Avenue \\ Cambridge, MA 02138 \\ December 2004
}

For helpful comments, we thank Andy Atkeson, Pierpaolo Battigalli, Alberto Bisin, V.V. Chari, Patrick Kehoe, Alessandro Lizzeri, Kiminori Matsuyama, Stephen Morris, Ivan Werning, and seminar participants at Berkeley, MIT, Northwestern, NYU, UBC, UCLA, Yale, the Minneapolis FRB, and the SED conference. The views expressed herein are those of the author(s) and do not necessarily reflect the views of the National Bureau of Economic Research.

(C) 2004 by George-Marios Angeletos, Christian Hellwig, and Alessandro Pavan. All rights reserved. Short sections of text, not to exceed two paragraphs, may be quoted without explicit permission provided that full credit, including $($ notice, is given to the source. 
Information Dynamics and Equilibrium Multiplicity in Global Games of Regime Change

George-Marios Angeletos, Christian Hellwig, and Alessandro Pavan

NBER Working Paper No. 11017

December 2004

JEL No. C7, D7, D8, F3

\begin{abstract}
Global games of regime change - that is, coordination games of incomplete information in which a status quo is abandoned once a sufficiently large fraction of agents attacks it - have been used to study crises phenomena such as currency attacks, bank runs, debt crises, and political change. We extend the static benchmark examined in the literature by allowing agents to accumulate information over time and take actions in many periods. It is shown that dynamics may lead to multiple equilibria under the same information assumptions that guarantee uniqueness in the static benchmark. Multiplicity originates in the interaction between the arrival of information over time and the endogenous change in beliefs induced by the knowledge that the regime survived past attacks. This interaction also generates interesting equilibrium properties, such as the possibility that fundamentals predict the eventual regime outcome but not the timing or the number of attacks, or that dynamics alternate between crises and phases of tranquility without changes in fundamentals.
\end{abstract}

George -Marios Angeletos

Department of Economics

MIT

50 Memorial Drive, E51-251

Cambridge, MA 02142

and NBER

angelet@mit.edu

Christian Hellwig

UCLA

chris@econ.ucla.edu

Alessandro Pavan

Northwestern University

alepavan@northwestern.edu 


\section{Introduction}

Coordination games in which a status quo is abandoned once a sufficiently large number of agents takes an action that favors regime change have been used to study a variety of socioeconomic phenomena. Whereas the earlier contributions in the literature focused on the existence and implications of multiple equilibria when agents can perfectly coordinate with each other, recent work on global games by Carlsson and van Damme (1993) and Morris and Shin (1998, 2000, 2001) has emphasized the fragility of this multiplicity to perturbations of the information structure away from common knowledge: a unique equilibrium often survives when agents observe a payoff-relevant variable, such as the strength of the status quo, with small idiosyncratic noise.

Variants of this uniqueness result have been established in the context of currency crises, bank runs, debt crises, and political change. ${ }^{1}$ Most of this work assumes a static coordination game, thus abstracting from the possibility that agents may have the option to take multiple shots against the regime. For many of the applications of interest, however, this possibility seems relevant.

In this paper, we extend the static benchmark examined in the literature by allowing agents to take actions in multiple periods and accumulate information over time. There is a large number of agents and two possible regimes, the status quo and an alternative. In any given period, each agent has a binary choice: he may either "attack" the status quo (that is, take an action that favors regime change) or "not attack". Attacking is individually optimal if and only if the probability of regime change in that period is sufficiently high. The status quo, in turn, is abandoned if and only if the fraction of agents attacking exceeds a critical value $\theta \in \mathbb{R}$, which parametrizes the strength of the status quo. $\theta$ is the component of the payoff structure - commonly referred to as the exogenous fundamentals - which is never common knowledge among the agents. Instead, as time passes, agents receive noisy private signals about $\theta$.

We show that dynamics may sustain multiple equilibria under the same information assumptions that guarantee uniqueness in the static benchmark, namely that the precision of the agents' private information is sufficiently high relative to the precision of the initial common prior. Multiplicity originates in the interaction between two elements: the knowledge that the regime survived past attacks and the arrival of new private information over time.

In the first period, agents find it dominant to attack for sufficiently low realizations of their private signals. This ensures that, in any equilibrium, regime change takes place in the first period for sufficiently low $\theta$. At any future date, the observation that the status quo is still in place makes it common certainty that it is not too weak, for otherwise it would have collapsed under the first attack.

\footnotetext{
${ }^{1}$ See Morris and Shin (1998) for currency crises; Goldstein and Pauzner (2000) and Rochet and Vives (2004) for bank runs; Morris and Shin (2004) and Corsetti, Guimaraes and Roubini (2004) for debt crises; Chamley (1999) for regime switches; Atkeson (2000) and Edmond (2004) for riots and political change.
} 
This effect implies that no agent is willing to attack in any subsequent period if he expects no other agent to attack. Hence, there always exists an equilibrium in which no attack occurs after the first period. In fact, if no new information were to arrive over time, this would be the unique equilibrium of the game. The possibility to take multiple shots against the regime would then add nothing to the static analysis.

However, as agents receive new private signals about $\theta$, the impact of the knowledge that the regime survived past attacks on posterior beliefs eventually vanishes. For the same reason, the dependence of posterior beliefs on the initial common prior also diminishes over time. When the prior is relatively aggressive, in the sense that it induces a large attack in the first period, the increase in the precision of private information would induce less aggressive behavior even in the absence of past attacks. In this case, no action after the first period remains the unique equilibrium. In contrast, when the prior is relatively lenient, both the discounting of the prior and the discounting of the knowledge that the regime survived past attacks contribute to making new attacks eventually possible. Hence, there also exist equilibria where agents take multiple shots against the regime. Indeed, we show that, unless the game ends for exogenous reasons in finite time, any arbitrary number of attacks can be sustained in equilibrium.

In the benchmark model, we deliberately assume away the possibility that the critical size of attack necessary for regime change varies over time. This allows us to isolate the effects of changes in information, as opposed to changes in fundamentals, on the dynamics of coordination. Nevertheless, we also show that the multiplicity result is robust to the introduction of shocks to the fundamentals - in which case dominant actions may exist in every period - provided that the volatility of these shocks is sufficiently small. In the limit, as the volatility vanishes, any equilibrium of the benchmark model can be approximated arbitrarily closely by an equilibrium in the game with shocks. What sustains the multiplicity of equilibria and the corresponding dynamics is again the combination of the arrival of new information over time with the endogenous upward shift in posterior beliefs that follows from the knowledge that the regime survived past attacks. That this shift takes the form of a truncation in the benchmark model simplifies the construction of the equilibrium set, but is not essential for the multiplicity result.

We also emphasize that multiplicity does not originate from the presence of exogenous public signals or the observation of the size of past attacks. Indeed, for most of the analysis we assume away any such source of information and concentrate on the case in which agents receive only private signals over time. What the introduction of public news does, is to "smooth out" the dependence of the determinacy of equilibria on the initial prior: multiple equilibria then exist whatever the horizon of the game and for any prior mean and any relative precision of public and private information. Finally, introducing endogenous signals about the size of past attacks - a simple form of (noisy) social learning - does not change the equilibrium dynamics, except for the 
possibility that the endogenous information revealed by the size of an attack may substitute for the arrival of new exogenous information and render further attacks possible immediately after an unsuccessful one.

The existence of multiple equilibria leads to dynamics that might not have been possible with either common knowledge or a unique equilibrium. For example, fundamentals may determine the final regime outcome (e.g., whether a currency is eventually devalued) but not the timing and number of attacks. Moreover, dynamics may take the form of sequences of periods in which attacks can not occur and agents only accumulate information, followed by periods in which an attack is possible but does not take place, eventually resulting in a new attack. An economy can thus transit from phases of tranquility to crises without any change in the underlying economic fundamentals.

Below, we discuss the relation of the paper to the pertinent literature. Section 2 then reviews the static benchmark and introduces the dynamic model. Section 3 characterizes the set of monotone equilibria. Section 4 establishes the multiplicity result and discusses equilibrium dynamics. Section 5 examines robustness to the introduction of shocks in the fundamentals. Section 6 introduces public news and signals about past attacks. Section 7 concludes. Proofs omitted in the main text are presented in the Appendix.

Related Literature. This paper contributes to a small but growing literature on dynamic global games. Heidhues and Melissas (2003) identify a condition of dynamic strategic complementarity that suffices for uniqueness in an investment model; this condition is also implicit in Giannitsarou and Toxvaerd's (2003) uniqueness theorem for recursive binary-action games. Dasgupta (2002) examines the role of social learning in a two-period investment model with irreversible actions. Levin (2001) considers a global game with overlapping generations of players. Goldstein and Pauzner (2001) and Goldstein (2002) consider models of contagion. Frankel and Pauzner (2000), on the other hand, consider a complete-information dynamic coordination game, where uniqueness is obtained by assuming aggregate shocks and idiosyncratic inertia. None of these papers, however, examines the role of information dynamics in games of regime change.

Closer to our analysis, Morris and Shin (1999) consider a dynamic model whose stage game is similar to ours, but where fundamentals follow a random walk and are commonly observed at the end of each period. This reduces the analysis to a sequence of static games with a unique equilibrium.

This paper departs from the above literature in that it considers dynamics as a natural source of information. In this respect, it shares with Angeletos, Hellwig and Pavan (2003) - which considers the signaling effects of policy in a static game - the idea that endogenous information structures may overturn uniqueness results in global games and lead to predictions that would have not been possible with either common knowledge or a unique equilibrium.

Finally, we share with Chari and Kehoe (2003) the motivation that understanding the dynamics 
of information may help understand the dynamics of crises. However, whereas they focus on herding, we examine the role of information for coordination.

\section{A simple game of regime change}

\subsection{Static benchmark}

Model set-up. There is a continuum of agents of measure one, indexed by $i$ and uniformly distributed over $[0,1]$. Agents move simultaneously, choosing between two actions: they can either attack the status quo (i.e., take an action that favors regime change) or refrain from attacking.

The payoff structure is illustrated in Table 1. The payoff from not attacking $\left(a_{i}=0\right)$ is zero, whereas the payoff from attacking $\left(a_{i}=1\right)$ is $1-c>0$ if the status quo is abandoned $(R=1)$ and $-c<0$ otherwise $(R=0)$, where $c \in(0,1)$ parametrizes the relative cost of attacking. An agent hence finds it optimal to attack if and only if he expects regime change with probability at least $c$. The status quo is in turn abandoned if and only if the measure of agents attacking, which we denote by $A$, is greater than or equal to $\theta \in \mathbb{R}$, which parametrizes the strength of the status quo. An agent's incentive to attack thus increases with the aggregate size of attack, implying that agents' actions are strategic complements. ${ }^{2}$

\begin{tabular}{|l|c|c|}
\hline & Regime Change $(A \geq \theta)$ & Status Quo $(A<\theta)$ \\
\hline Attack $\left(a_{i}=1\right)$ & $1-c$ & $-c$ \\
\hline Not Attack $\left(a_{i}=0\right)$ & 0 & 0 \\
\hline
\end{tabular}

Table 1. Payoffs

Agents have heterogeneous information about the strength of the status quo. Nature first draws $\theta$ from a normal distribution $\mathcal{N}(z, 1 / \beta)$, which defines the initial common prior about $\theta$. Each agent then receives a private signal $x_{i}=\theta+\xi_{i}$, where $\xi_{i} \sim \mathcal{N}(0,1 / \alpha)$ is noise, i.i.d. across agents and independent of $\theta$. The Normality assumptions allow to parametrize the information structure parsimoniously with $(\alpha, \beta, z)$, that is, the precision of private information and the precision and mean of the common prior.

Interpretation. Although the game presented above is highly stylized, it admits a variety of interpretations and possible applications. The most celebrated examples are self-fulfilling bank runs, currency attacks, and debt crises. In these contexts, regime change occurs, respectively, when a large run forces the banking system to suspend its payments, when a large speculative attack

\footnotetext{
${ }^{2}$ The role of coordination is most evident when $\theta$ is commonly known by all agents: for $\theta \in(0,1]$, there exist two pure-strategy equilibria, one in which all agents attack and the status quo is abandoned $(A=1 \geq \theta)$ and another in which no agent attacks and the status quo is maintained $(A=0<\theta)$.
} 
forces the central bank to abandon the peg, or when a country/company fails to coordinate its creditors and is forced to bankruptcy. The model can also be interpreted as one of political change, in which a large number of citizens decide whether or not to take actions to subvert a repressive dictator or some other political establishment. ${ }^{3}$

Equilibrium analysis. Note that the c.d.f. of an agent's posterior about $\theta$ is decreasing in his private signal $x$. Moreover, it is strictly dominant to attack for sufficiently low signals - namely for $x<\underline{x}$, where $\underline{x}$ solves $\operatorname{Pr}(\theta \leq 0 \mid \underline{x})=c$ - and not to attack for sufficient high signals - namely for $x>\bar{x}$, where $\bar{x}$ solves $\operatorname{Pr}(\theta \leq 1 \mid \bar{x})=c$. It is thus natural to look at monotone Bayesian Nash equilibria in which the agents' strategy is non-increasing in $x$.

Indeed, suppose there is a threshold $\hat{x} \in \mathbb{R}$ such that each agent attacks if and only if $x \leq \hat{x}$. The measure of agents attacking is then decreasing in $\theta$ and is given by $A(\theta)=\operatorname{Pr}(x \leq \hat{x} \mid \theta)=$ $\Phi(\sqrt{\alpha}(\hat{x}-\theta))$, where $\Phi$ is the c.d.f. of the standard Normal. It follows that the status quo is abandoned if and only if $\theta \leq \hat{\theta}$, where $\hat{\theta}$ solves $\hat{\theta}=A(\hat{\theta})$, or equivalently

$$
\hat{\theta}=\Phi(\sqrt{\alpha}(\hat{x}-\hat{\theta})) .
$$

The posterior probability of regime change for an agent with signal $x$ is then simply $\operatorname{Pr}(R=1 \mid x)=$ $\operatorname{Pr}(\theta \leq \hat{\theta} \mid x)$. Since the latter is decreasing in $x$, each agent finds it optimal to attack if and only if $x \leq \hat{x}$, where $\hat{x}$ solves $\operatorname{Pr}(\theta \leq \hat{\theta} \mid \hat{x})=c$, or equivalently

$$
\Phi\left(\sqrt{\alpha+\beta}\left(\hat{\theta}-\frac{\alpha}{\alpha+\beta} \hat{x}-\frac{\beta}{\alpha+\beta} z\right)\right)=c .
$$

A monotone equilibrium is thus identified by a joint solution $(\hat{x}, \hat{\theta})$ to $(1)$ and $(2)$. Such a solution always exists and is unique for all $z$ if and only if $\alpha \geq \beta^{2} /(2 \pi)$. Moreover, iterated elimination of strictly dominated strategies implies that, when the monotone equilibrium is unique, there is no other equilibrium. We conclude that

Proposition 1 (Static benchmark) In the static game, the equilibrium is unique if and only if $\alpha \geq \beta^{2} /(2 \pi)$, and is in monotone strategies.

\subsection{Dynamic game}

We modify the static game reviewed above in two ways: first, we allow agents to attack the status quo repeatedly; second, we let agents accumulate information over time.

Time is discrete and indexed by $t \in\{1,2, \ldots\}$. The game continues as long as the status quo is in place and is over once the status quo is abandoned. We denote with $R_{t}=0$ the event that the status quo is still in place at the beginning of period $t$, with $R_{t}=1$ the alternative event, with $a_{i t} \in\{0,1\}$ the action of agent $i$, and with $A_{t} \in[0,1]$ the measure of agents attacking in period

\footnotetext{
${ }^{3}$ For references, see footnote 1 .
} 
$t$. Conditional on the regime being in place at the beginning of period $t\left(R_{t}=0\right)$, the regime is abandoned in that period $\left(R_{t+1}=1\right)$ if and only if $A_{t} \geq \theta$, where $\theta$ again represents the strength of the status quo. Agent $i$ 's payoff in period $t$ is

$$
\pi_{i t}=a_{i t}\left(R_{t+1}-c\right)
$$

and his payoff from the entire game is $\Pi_{i}=\sum_{t=1}^{\infty} \rho^{t-1}\left(1-R_{t}\right) \pi_{i t}$, where $\rho \in(0,1)$ is the discount factor.

Like in the static model, $\theta$ is drawn at the beginning of the game from $\mathcal{N}(z, 1 / \beta)$, which defines the initial common prior, and never becomes common knowledge. Private information, however, evolves over time. In each period $t \geq 1$, every agent $i$ receives a private signal $\tilde{x}_{i t}=\theta+\xi_{i t}$ about $\theta$, where $\xi_{i t} \sim \mathcal{N}\left(0,1 / \eta_{t}\right)$ is i.i.d. across $i$, independent of $\theta$, and serially uncorrelated. Let $\tilde{x}_{i}^{t}=\left\{\tilde{x}_{i \tau}\right\}_{\tau=1}^{t}$ denote agent $i$ 's history of private signals up to period $t$. Individual actions and the size of past attacks are not observable, ${ }^{4}$ hence the public history in period $t$ simply consists of the information that the regime is still in place, whereas the private history of an agent is the sequence of own private signals $\tilde{x}_{i}^{t}$ and own past actions. Finally, we let $\alpha_{t} \equiv \sum_{\tau=1}^{t} \eta_{\tau}$ and assume that

$$
\infty>\alpha_{t} \geq \beta^{2} /(2 \pi) \forall t \quad \text { and } \quad \lim _{t \rightarrow \infty} \alpha_{t}=\infty
$$

As shown in the next section, $\alpha_{t}$ parametrizes the precision of private information in period $t$. The restrictions above ensure equilibrium uniqueness in the static game with precision $\alpha_{t}$, and that private information becomes infinitely precise only in the limit.

Remark. While this dynamic game is highly stylized, it captures two important dimensions that are absent in the static benchmark: first, the possibility of multiple attacks against the status quo; and, second, the evolution of beliefs about the probability of regime change. By assuming that per-period payoffs do not depend on past or future actions and by ignoring specific institutional details, the model may of course fail to capture other interesting effects introduced by dynamics, such as, for example, the role of wealth accumulation or liquidity in currency crises. However, abstracting from these other dimensions allows us to isolate information as the driving force for the dynamics of coordination and crises.

Equilibrium. In what follows, we limit attention to monotone equilibria, that is, symmetric Perfect Bayesian equilibria in which the probability an agent attacks in period $t$, which with a slight abuse of notation we denote with $a_{t}\left(\tilde{x}^{t}\right)$, is non-increasing in his private signals $\tilde{x}^{t}$ and independent of his own past actions. ${ }^{5}$ Restricting attention to this class of equilibria suffices to establish our multiplicity results.

\footnotetext{
${ }^{4}$ The possibility that agents observe noisy signals about aggregate past activity is considered in Section 6 .

${ }^{5}$ We do not restrict the set of available strategies: we look at equilibria in which these properties are satisfied.
} 


\section{Equilibrium characterization}

Since neither individual nor aggregate actions are observable, and $R_{t}=0$ is always compatible with any strategy profile at any $t,{ }^{6}$ no agent can detect out-of-equilibrium play as long as the status quo is in place. It follows that beliefs are pinned down by Bayes' rule in any relevant history of the game. Furthermore, payoffs in one period do not depend on own or other players' actions in any other period and hence strategies are sequentially rational if and only if they maximize period-by-period payoffs. Finally, define $x_{t}$ and $\alpha_{t}$ recursively by

$$
x_{t}=\frac{\alpha_{t-1}}{\alpha_{t}} x_{t-1}+\frac{\eta_{t}}{\alpha_{t}} \tilde{x}_{t} \quad \text { and } \quad \alpha_{t}=\alpha_{t-1}+\eta_{t}
$$

with $x_{1}=\tilde{x}_{1}$ and $\alpha_{1}=\eta_{1}$; and note that $x_{t}$ is a sufficient statistic for the agent's private information $\tilde{x}^{t}=\left\{\tilde{x}_{\tau}\right\}_{\tau=1}^{t}$ with respect to $\theta$ and therefore with respect to regime change as well. We conclude that, in any monotone equilibrium,

$$
a_{t}\left(\tilde{x}^{t}\right) \in \arg \max _{a \in[0,1]}\left\{\left(\operatorname{Pr}\left[R_{t+1}=1 \mid x_{t}, R_{t}=0\right]-c\right) a\right\},
$$

where $\operatorname{Pr}[\cdot]$ is pinned down by Bayes' rule using $\left\{a_{t}(\cdot)\right\}_{t=1}^{\infty}$.

This also implies that, in any equilibrium of the dynamic game, agents play in period 1 exactly as in the static game in which they can attack only in that period: an agent attacks if and only if his private signal is sufficiently low and the status quo is maintained if and only if $\theta$ is sufficiently high. The following lemma shows that a similar property applies to subsequent periods. ${ }^{7}$

Lemma 1 For any monotone equilibrium, there is a sequence $\left\{x_{t}^{*}, \theta_{t}^{*}\right\}_{t=1}^{\infty}$, where $x_{t}^{*} \in \overline{\mathbb{R}} \equiv \mathbb{R} \cup$ $\{ \pm \infty\}$ and $\theta_{t-1}^{*} \in(0,1)$, such that:

(i) at any $t \geq 1$, an agent attacks if $x_{t}<x_{t}^{*}$ and does not attack if $x_{t}>x_{t}^{*}$;

(ii) the status quo is in place at any $t \geq 2$ if and only if $\theta>\theta_{t-1}^{*}$.

The fact that the status quo is in place in period $t \geq 2$ makes it common certainty that $\theta>\theta_{t-1}^{*}$. Since $\theta_{t-1}^{*} \geq \theta_{1}^{*}>0$, this implies that there always exist equilibria in which nobody attacks in period $t \geq 2$, in which case $x_{t}^{*}=-\infty$ and $\theta_{t}^{*}=\theta_{t-1}^{*}$. In particular, there exists an equilibrium in which an attack takes place in period 1 and never after. If this were always the unique equilibrium, the possibility to take repeated actions against the regime would add nothing to the static analysis and the equilibrium outcome in the dynamic game would coincide with that in the static benchmark. In what follows we thus examine under what conditions there also exist equilibria with further attacks.

\footnotetext{
${ }^{6}$ Indeed, the regime always survives any attack for $\theta>1$ and no realization of the private signal rules out $\theta>1$.

${ }^{7}$ To simplify the notation, we allow for $x_{t}^{*}=-\infty$ and $x_{t}^{*}=+\infty$, with which we denote the case where an agent attacks for, respectively, none or every realization of his private information.
} 
By Lemma 1, the size of the attack is given by $A_{t}(\theta)=\operatorname{Pr}\left(x \leq x_{t}^{*} \mid \theta\right)$, which is decreasing in $\theta$, and the probability of regime change for an agent with sufficient statistic $x_{t}$ is $\operatorname{Pr}\left(R_{t+1}=\right.$ $\left.1 \mid x_{t}, R_{t}=0\right)=\operatorname{Pr}\left(\theta \leq \theta_{t}^{*} \mid x_{t}, \theta>\theta_{t-1}^{*}\right)$, which is decreasing in $x_{t}$ if $\theta_{t}^{*}>\theta_{t-1}^{*}$. It follows that, in any equilibrium in which an attack occurs in period $t, \theta_{t}^{*}$ and $x_{t}^{*}$ solve

$$
\begin{gathered}
\theta_{t}^{*}=\Phi\left(\sqrt{\alpha_{t}}\left(x_{t}^{*}-\theta_{t}^{*}\right)\right) \\
1-\frac{\Phi\left(\sqrt{\alpha_{t}+\beta}\left(\frac{\alpha}{\alpha_{t}+\beta} x_{t}^{*}+\frac{\beta}{\alpha_{t}+\beta} z-\theta_{t}^{*}\right)\right)}{\Phi\left(\sqrt{\alpha_{t}+\beta}\left(\frac{\alpha}{\alpha_{t}+\beta} x_{t}^{*}+\frac{\beta}{\alpha_{t}+\beta} z-\theta_{t-1}^{*}\right)\right)}=c .
\end{gathered}
$$

Conditions (4) and (5) are the analogs in the dynamic game of conditions (1) and (2) in the static game: (4) states that the equilibrium size of an attack is equal to the critical size that triggers regime change if and only if the fundamentals are $\theta_{t}^{*}$, while (5) states that an agent is indifferent between attacking and not attacking if and only if his private information is $x_{t}^{*}$.

Solving (4) for $x_{t}^{*}$ gives $x_{t}^{*}=X\left(\theta_{t}^{*}, \alpha_{t}\right)$ and substituting this into (5) gives a single equation in $\theta_{t}^{*}$, namely

$$
U\left(\theta_{t}^{*}, \theta_{t-1}^{*}, \alpha_{t}, \beta, z\right)=0
$$

where $X:[0,1] \times \mathbb{R}_{+} \rightarrow \overline{\mathbb{R}}$ and $U:[0,1] \times \overline{\mathbb{R}} \times \mathbb{R}_{+}^{2} \times \mathbb{R} \rightarrow[-c, 1-c]$ are defined as follows: ${ }^{8}$

$$
\begin{aligned}
& X\left(\theta^{*}, \alpha\right) \equiv \theta^{*}+\frac{1}{\sqrt{\alpha}} \Phi^{-1}\left(\theta^{*}\right) \\
& U\left(\theta^{*}, \theta_{-1}, \alpha, \beta, z\right) \equiv \begin{cases}1-c & \text { if } \theta^{*}=0>\theta_{-1} \\
1-\frac{\Phi\left(\frac{\sqrt{\alpha}}{\sqrt{\alpha+\beta}}\left[\Phi^{-1}\left(\theta^{*}\right)+\frac{\beta}{\sqrt{\alpha}}\left(z-\theta^{*}\right)\right]\right)}{\Phi\left(\frac{\sqrt{\alpha}}{\sqrt{\alpha+\beta}}\left[\Phi^{-1}\left(\theta^{*}\right)+\frac{\beta}{\sqrt{\alpha}}\left(z-\theta^{*}\right)\right]+\sqrt{\alpha+\beta}\left(\theta^{*}-\theta_{-1}\right)\right)}-c & \text { if } \theta^{*}>\max \left\{0, \theta_{-1}\right\} \\
-c & \text { if } \theta^{*} \leq \theta_{-1}\end{cases}
\end{aligned}
$$

The functions $X$ and $U$ have a simple interpretation: $X\left(\theta^{*} ; \alpha\right)$ is the "marginal agent" that implements regime change for $\theta \leq \theta^{*}$ when the precision of private information is $\alpha$ (that is, the threshold $x^{*}$ such that, if agents attack if and only if $x \leq x^{*}$, the status quo is abandoned if and only if $\left.\theta \leq \theta^{*}\right) ; U\left(\theta^{*}, \theta_{-1}, \alpha, \beta, z\right)$ is the expected net payoff of the marginal agent from attacking, conditional on the knowledge that $\theta>\theta_{-1}$. Condition (6) thus simply requires that the marginal agent is indifferent between attacking and not attacking in period $t \geq 2$. As for $t=1$, since the regime has never been challenged in the past, the corresponding indifference condition is $U\left(\theta_{1}^{*},-\infty, \alpha_{1}, \beta, z\right)=0$, where $U(\theta,-\infty, \alpha, \beta, z)$ coincides with the payoff of the marginal agent in the static benchmark.

The next proposition then provides the complete set of necessary and sufficient conditions for monotone equilibria.

\footnotetext{
${ }^{8}$ With a slight abuse of notation, we let $\Phi(+\infty)=1, \Phi(-\infty)=0, \Phi^{-1}(1)=\infty$ and $\Phi^{-1}(0)=-\infty$.
} 
Proposition 2 (Equilibrium characterization) $\left\{a_{t}(\cdot)\right\}_{t=1}^{\infty}$ is a monotone equilibrium if and only if there exists a sequence $\left\{x_{t}^{*}, \theta_{t}^{*}\right\}_{t=1}^{\infty}$ such that:

(i) for all $t, a_{t}(\cdot)=1$ if $x_{t}<x_{t}^{*}$ and $a_{t}(\cdot)=0$ if $x_{t}>x_{t}^{*}$.

(ii) for $t=1, \theta_{1}^{*}$ solves $U\left(\theta_{1}^{*},-\infty, \alpha_{1}, \beta, z\right)=0$ and $x_{1}^{*}=X\left(\theta_{1}^{*}, \alpha_{1}\right)$.

(iii) for any $t \geq 2$, either $\theta_{t}^{*}=\theta_{t-1}^{*}>0$ and $x_{t}^{*}=-\infty$, or $\theta_{t}^{*}>\theta_{t-1}^{*}$ is a solution to $U\left(\theta_{t}^{*}, \theta_{t-1}^{*}, \alpha_{t}, \beta, z\right)=0$ and $x_{t}^{*}=X\left(\theta_{t}^{*}, \alpha_{t}\right)$.

An equilibrium always exists.

Proposition 2 provides a simple algorithm for constructing the entire set of monotone equilibria: start with $t=1$ and let $\theta_{1}^{*}$ be the unique solution to $U\left(\theta_{1}^{*},-\infty, \alpha_{1}, \beta, z\right)=0$; proceed to period $t=2$ and let either $\theta_{2}^{*}=\theta_{1}^{*}$ or $\theta_{2}^{*}$ be a solution to $(6)$; repeat the same step for all $t \geq 3$; the set of sequences $\left\{\theta_{t}^{*}\right\}_{t=1}^{\infty}$ constructed this way, together with the associated sequences $\left\{x_{t}^{*}\right\}_{t=1}^{\infty}$, gives the set of monotone equilibria. Note that the above characterization is independent of whether the horizon is finite or infinite: it is clearly valid even if the game ends exogenously at an arbitrary period $T<\infty$.

Existence of equilibrium follows immediately from the fact that $U\left(\theta_{1}^{*},-\infty, \alpha_{1}, \beta, z\right)=0$ always admits a solution and hence $\theta_{t}^{*}=\theta_{1}^{*}$ for all $t$ is always an equilibrium. To understand whether there are other equilibria, the next lemma investigates the properties of $U$ and the existence of solutions to $(6)$.

Lemma 2 (i) $U\left(\theta^{*}, \theta_{-1}, \alpha, \beta, z\right)$ is continuous in all its arguments, non-monotonic and singlepeaked in $\theta^{*}$ when $\theta_{-1} \in(0,1)$, and strictly decreasing in $\theta_{-1}$ and $z$ for $\theta_{-1}<\theta^{*}$. Furthermore, for all $\theta_{-1}<1$ and $\theta^{*}>\theta_{-1}, \lim _{\alpha \rightarrow \infty} U\left(\theta^{*}, \theta_{-1}, \cdot\right)=\theta_{\infty}-\theta^{*}$, where $\theta_{\infty} \equiv 1-c$.

(ii) Let $\hat{\theta}_{t}$ be the unique solution to $U\left(\hat{\theta}_{t},-\infty, \alpha_{t}, \beta, z\right)=0$. A solution to (6) exists only if $\theta_{t-1}^{*}<\hat{\theta}_{t}$ and is necessarily bounded from above by $\hat{\theta}_{t}$.

(iii) If $\theta_{t-1}^{*}>\theta_{\infty}$, a solution to (6) does not exist for $\alpha_{t}$ sufficiently high.

(iv) If $\theta_{t-1}^{*}<\theta_{\infty}$, a solution to (6) necessarily exists for $\alpha_{t}$ sufficiently high.

(v) If $\theta_{t-1}^{*}$ is the highest solution to (6) for period $t-1$, there exists $\underline{\alpha}>\alpha_{t-1}$ such that (6) admits no solution for any period $\tau \geq t$ such that $\alpha_{\tau}<\underline{\alpha}$.

The non-monotonicity of $U\left(\theta^{*}, \theta_{t-1}^{*}, \alpha_{t}, \beta, z\right)$ with respect to $\theta^{*}$ in any period $t \geq 2$ (where $\left.0<\theta_{t-1}^{*}<1\right)$ is a direct consequence of the fact that, for $\theta^{*}<\theta_{t-1}^{*}$, the marginal agent attaches probability zero to regime change. For $\theta^{*}>\theta_{t-1}^{*}$, on the other hand, his belief about regime change is necessarily positive, but converges to zero as $\theta^{*} \rightarrow 1$, for then $X\left(\theta^{*}, \alpha_{t}\right) \rightarrow \infty$ and hence he attaches probability one to $\theta>1$. His payoff is thus initially increasing and then decreasing, as illustrated by the solid line in Figure 1. Any intersection with the horizontal axis corresponds to a solution to (6). The dashed line instead represents the payoff of the marginal agent in the static 


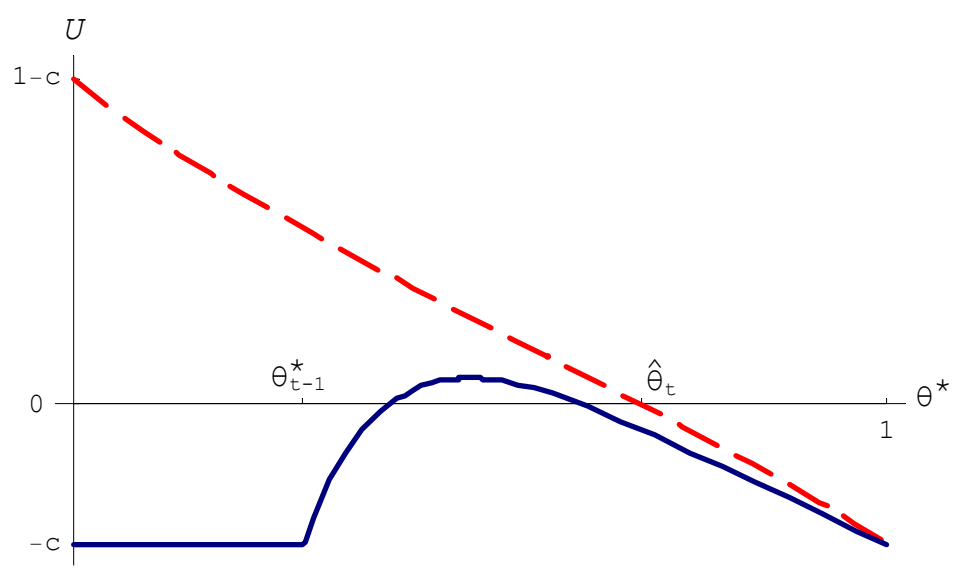

Figure 1: The payoff of the marginal agent.

game. Whereas the monotonicity of the latter ensures uniqueness, the non-monotonicity in the dynamic game leaves open the possibility for multiple equilibria.

The other properties identified in Lemma 2 are also quite intuitive. An increase in the mean of the common prior implies a first-order stochastic-dominance change in the posterior beliefs about $\theta$, which explains why $U$ decreases with $z$. To understand why $U$ is also decreasing in $\theta_{-1}$, note that the more aggressive the attacks in the past, the stronger the regime must have been in order to have survived these attacks, and therefore the lower the expected payoff from attacking in the current period.

For the same reason, at any $t \geq 2$, the payoff of the marginal agent is always lower than $U\left(\theta^{*},-\infty, \alpha_{t}, \beta, z\right)$, that is, the payoff in the static game where the precision of private information is $\alpha_{t}$. This explains why the static-game threshold $\hat{\theta}_{t}$ (which corresponds to the intersection of the dashed line with the horizontal axis in Figure 1) is an upper bound for any solution to (6). Nevertheless, the impact of the information that $\theta>\theta_{t-1}^{*}$ on posterior beliefs vanishes for any $x_{t}>\theta_{t-1}^{*}$ as $\alpha_{t} \rightarrow \infty$, and hence, for any $\theta^{*}>\theta_{t-1}^{*}$, the difference between $U\left(\theta^{*}, \theta_{t-1}^{*}, \alpha_{t}, \beta, z\right)$ and $U\left(\theta^{*},-\infty, \alpha_{t}, \beta, z\right)$ also vanishes. Combined with the fact that $U\left(\theta^{*},-\infty, \alpha_{t}, \beta, z\right) \rightarrow \theta_{\infty}-\theta^{*}$ as $\alpha_{t} \rightarrow \infty$, this in turn implies that, for $\alpha_{t}$ sufficiently high, (6) admits a solution if and only if $\theta_{t-1}^{*}<\theta_{\infty}$, where $\theta_{\infty}$ is the limit of the equilibrium threshold of the static game as the precision of private information becomes infinite (i.e., $\theta_{\infty}=\lim _{t \rightarrow \infty} \hat{\theta}_{t}$ ).

Finally, to understand $(v)$, note that any unsuccessful attack causes an upward shift (a firstorder stochastic-dominance change) in the posterior beliefs about the strength of the regime, which other things equal reduces the expected payoff from attacking. It follows that, if the largest possible attack (that is, the highest solution of (6)) is played in one period and no new information arrives thereafter, no attack is possible in any subsequent period. By continuity then, further attacks remain impossible as long as the change in the precision of private information is not large enough. 


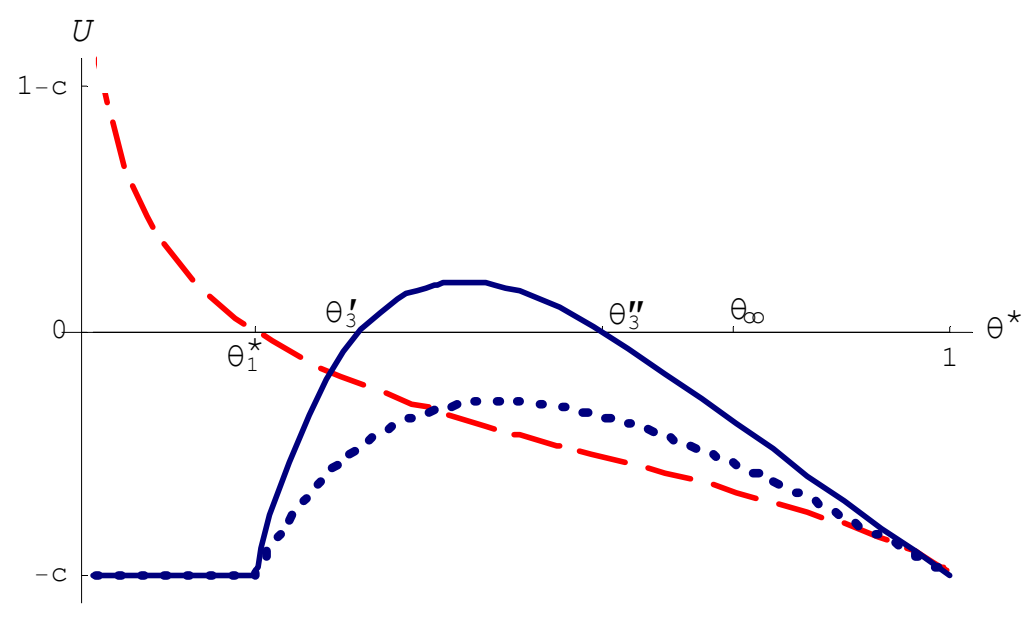

Figure 2: Equilibria with multiple attacks.

\section{Multiplicity and dynamics}

Part $(v)$ of Lemma 2 highlights that the arrival of new private information is necessary for further attacks to become possible after period 1. Whether this is also sufficient depends on the initial prior. This is because an increase in the precision of private information leads agents to discount, not only the information conveyed by the fact that the regime survived past attacks, but also their initial prior beliefs.

When $z$ is low ("aggressive prior"), an increase in the precision of private information makes the marginal agent less aggressive in the static game (that is, $\hat{\theta}_{t}$ decreases with $\alpha_{t}$ ). The knowledge that the regime survived past attacks then only reinforces this effect. Therefore, for $z$ sufficiently low, there exists a unique equilibrium and is such that no attack ever occurs after the first period.

When instead $z$ is high ("lenient prior"), the arrival of new private information makes the marginal agent more aggressive, and may eventually offset the incentive not to attack induced by the knowledge that the regime survived past attacks. Indeed, if $z$ is high enough so that $\theta_{1}^{*}<\theta_{\infty}$, Lemma 2 ensures that a second attack necessarily becomes possible once $\alpha_{t}$ is sufficiently high. Such an example is illustrated in Figure 2. The dashed line represents the payoff of the marginal agent in period 1 . Its intersection with the horizontal axis defines $\theta_{1}^{*}<\theta_{\infty}$. The payoff of the marginal agent in period 2 is represented by the dotted line, and that in period 3 by the solid line. Clearly, $\alpha_{2}$ is low enough that no attack is possible in period 2. In contrast, $\alpha_{3}$ is high enough that a new attack is possible. There thus exist at least three equilibria in this example: one in which $\theta_{t}^{*}=\theta_{1}^{*}$ for all $t$, another in which $\theta_{2}^{*}=\theta_{1}^{*}$ and $\theta_{t}^{*}=\theta_{3}^{\prime}$ for all $t \geq 3$, and a third in which $\theta_{2}^{*}=\theta_{1}^{*}$ and $\theta_{t}^{*}=\theta_{3}^{\prime \prime}$ for all $t \geq 3$, where $\theta_{3}^{\prime}$ and $\theta_{3}^{\prime \prime}$ correspond to the two intersections of the solid line with the horizontal axis. 
In the example of Figure 2, both $\theta_{3}^{\prime}$ and $\theta_{3}^{\prime \prime}$ are lower than $\theta_{\infty}$. By Lemma 2, then, a third attack also becomes possible at some future date. More generally, if $z$ is sufficiently high, any solution to $(6)$ is bounded from above by $\theta_{\infty}$ in all periods, which ensures that a new attack eventually becomes possible after any unsuccessful one. Hence, for $z$ sufficiently high, not only there are multiple equilibria, but any arbitrary number of attacks can be sustained in equilibrium.

Theorem 1 (Multiplicity) There exist thresholds $\underline{z} \leq \bar{z} \leq \overline{\bar{z}}$ such that:

(i) If $z \leq \underline{z}$, there is a unique monotone equilibrium and an attack occurs only in period one.

(ii) If $z \in(\underline{z}, \bar{z})$, there are at most finitely many monotone equilibria and there exists $\bar{t}<\infty$ such that no attack occurs after period $\bar{t}$.

(iii) If $z>\bar{z}$, there are infinitely many equilibria; if in addition $z>\overline{\bar{z}}$, for any $t$ and $N$, there is an equilibrium in which $N$ attacks occur after period $t$.

Finally, $\underline{z}=\bar{z}=\overline{\bar{z}}$ when $c \leq 1 / 2$, whereas $\underline{z} \leq \bar{z}<\overline{\bar{z}}$ when $c>1 / 2$.

Proof. Recall that $\theta_{1}^{*}=\hat{\theta}_{1}$ and, for all $t \geq 2, \theta_{t}^{*}<\hat{\theta}_{t}$, where $\hat{\theta}_{t}=\hat{\theta}\left(\alpha_{t}, \beta, z\right)$ is the unique solution to $U\left(\hat{\theta},-\infty, \alpha_{t}, \beta, z\right)=0$. As we show in Lemma A1 in the Appendix, there exist thresholds $\underline{z} \leq \bar{z} \leq \overline{\bar{z}}$ (possibly functions of $\alpha_{1}$ and $\beta$ ) with the following properties: $\hat{\theta}_{t} \leq \hat{\theta}_{1}$ for all $t$ if $z \leq \underline{z}$; $\hat{\theta}_{1} \leq(\geq) \theta_{\infty}$ if and only $z \geq(\leq) \bar{z}$; and $\hat{\theta}_{t}<\theta_{\infty}$ for all $t$ if and only if $z>\overline{\bar{z}}$.

(i) Consider first $z \leq \underline{z}$. Then, $\hat{\theta}_{t} \leq \hat{\theta}_{1}=\theta_{1}^{*}$ for all $t$, and hence, by part (ii) of Lemma 2, (6) admits no solution at any $t \geq 2$. The unique monotone equilibrium is thus $\theta_{t}^{*}=\theta_{1}^{*}$ for all $t$.

(ii) Next, consider $z \in(\underline{z}, \bar{z})$, in which case $\hat{\theta}_{1}=\theta_{1}^{*}>\theta_{\infty}$, but we can not rule out the possibility that there exists a period $t \geq 2$ such that $\hat{\theta}_{t}>\hat{\theta}_{1}$ and $U\left(\theta^{*}, \theta_{1}^{*}, \alpha_{t}, \beta, z\right)=0$ admits a solution. Nevertheless, since $\theta_{t-1}^{*} \geq \theta_{1}^{*}>\theta_{\infty}$ for all $t$, by part (iii) of Lemma 2 and the fact that $\alpha_{t} \rightarrow \infty$ as $t \rightarrow \infty$, there exists $\bar{t}<\infty$ such that (6) admits no solution for $t \geq \bar{t}$. Moreover, since $U$ is single-peaked, (6) has at most two solutions for $t<\bar{t}$. Hence, there are at most finitely many monotone equilibria, and in any equilibrium no attack occurs after period $\bar{t}$.

(iii) Finally, consider $z>\bar{z}$, in which case $\theta_{1}^{*}<\theta_{\infty}$. Then, by part (iv) of Lemma 2 , there exists a $t^{\prime}<\infty$ such that $U\left(\theta^{*}, \theta_{1}^{*}, \alpha_{t}, \beta, z\right)=0$ admits a solution for all $t \geq t^{\prime}$. Hence, for any $t \geq t^{\prime}$, there is an equilibrium in which $\theta_{\tau}^{*}=\theta_{1}^{*}$ for $\tau<t, \theta_{t}^{*}$ solves $U\left(\theta_{t}^{*}, \theta_{1}^{*}, \alpha_{t}, \beta, z\right)=0$, and $\theta_{\tau}^{*}=\theta_{t}^{*}$ for all $\tau>t$. That is, there exist (countably) infinitely many equilibria, indexed by the time at which the second attack occurs.

When $z \in(\bar{z}, \overline{\bar{z}})$, the second attack may lead to a threshold $\theta_{t}^{*}>\theta_{\infty}$, in which case a third attack might be impossible. If however $z>\overline{\bar{z}}$, then $\hat{\theta}_{t}<\theta_{\infty}$ for all $t$, and hence by part (ii) of Lemma $2, \theta_{t}^{*}<\theta_{\infty}$, for all $t$. But then by part (iv), a new attack eventually becomes possible after any unsuccessful one. It follows that, for any $t \geq 1$ and any $N \geq 1$, there exist increasing sequences $\left\{t_{2}, \ldots, t_{N}\right\}$ and $\left\{\theta_{2}, \ldots, \theta_{N}\right\}$, with $t_{2} \geq t$, such that $U\left(\theta_{2}, \theta_{1}^{*}, \alpha_{t_{2}}, \beta, z\right)=0, U\left(\theta_{3}, \theta_{2}, \alpha_{t_{3}}, \beta, z\right)=0$, and so on. The following is then an equilibrium: $\theta_{\tau}^{*}=\theta_{1}^{*}$ for $\tau<t_{2}, \theta_{\tau}^{*}=\theta_{j}$ for $\tau \in\left\{t_{j}, \ldots, t_{j+1}-1\right\}$ 
and $j \in\{2, \ldots, N-1\}$, and $\theta_{\tau}^{*}=\theta_{N}$ for $\tau \geq t_{N}$. That is, for any $t \geq 1$ and any $N \geq 1$, there exists an equilibrium in which $N$ attacks occur after date $t$.

The existence of infinitely many equilibria in the case $z>\bar{z}$ relies on the assumption that the game continues forever as long as the status quo is in place: if the game ended for exogenous reasons at a finite date, there would exist only finitely many equilibria. Nevertheless, as long as $z>\bar{z}$ and $\alpha_{t} \rightarrow \infty$ as $t \rightarrow \infty$, then, for any $M$, there exists a finite $T$ such that the game would have at least $M$ equilibria if it ended at date $T$. Moreover, even if $T=2, \alpha_{2}$ high enough suffices for the game to have multiple equilibria when $z>\bar{z}$. Finally, as we show in Section 6.1, the introduction of public signals ensures the existence of multiple equilibria for any $T \geq 2$ and any $z$.

In the remainder of this section, we identify equilibrium properties that may turn useful in understanding crises phenomena.

Corollary 1 Suppose $\theta>\theta_{\infty}$ and $z>\overline{\bar{z}}$. The status quo survives in any equilibrium. Nevertheless, there exists $\underline{t}<\infty$ such that, at any $t \geq \underline{t}$, an attack is possible, yet need not take place. Furthermore, any arbitrary number of attacks can occur.

This seems to square well with the common view that economic fundamentals may help predict eventual outcomes (e.g., whether a currency is eventually devalued) but not when a crisis will occur or whether attacks will cease. On the contrary, this view is inconsistent with the common-knowledge version of the model, in which case fundamentals fail to predict both the timing of attacks and the eventual regime outcome, ${ }^{9}$ as well as with unique-equilibrium models like Morris and Shin (1999), in which both the timing of attacks and the ultimate fate of the regime are uniquely pinned down by fundamentals.

Consider now how the dynamics of attacks depend on the dynamics of information.

Corollary 2 After the most aggressive attack for a given period occurs, the game enters a phase of tranquillity, during which no attack is possible. This phase is longer the slower the arrival of private information.

Along with the property that for $\theta>\theta_{\infty}$ and $z>\overline{\bar{z}}$ a new attack eventually becomes possible after any unsuccessful one, the above result implies that dynamics may take the form of cycles in which the economy alternates from phases of tranquillity to periods of crisis, eventually resulting into a new attack, without any change in the underlying fundamentals. Once again, this would not be possible either under common knowledge or with a unique equilibrium.

\footnotetext{
${ }^{9}$ Of course, this is true provided $\theta \in[0,1]$. If instead $\theta \notin[0,1]$, fundamentals predict both the timing and the final outcome, as for $\theta<0$ it is dominant to attack immediately and for $\theta>1$ it is dominant never to attack.
} 


\section{Unobservable shocks}

In this section, we examine the possibility that the critical size of attack that triggers regime change varies stochastically over time due to unobservable shocks. This is not only a realistic scenario, but also an appropriate perturbation. In the model without shocks, the knowledge that the regime is in place at $t \geq 2$ makes it common certainty that $\theta>0$, which explains why there always exist equilibria in which nobody attacks at $t \geq 2$. In contrast, shocks may guarantee that a positive measure of agents attack in every period by "smoothing out" the information generated by the knowledge that the regime survived past attacks and ensuring the existence of a lower dominance region in every period.

We modify the game as follows. Agents continue to receive private signals about $\theta$ as in the benchmark model, but the regime is now abandoned in period $t$ if and only if $A_{t} \geq \theta+\delta \omega_{t}$, where $\delta \geq 0$ and $\omega_{t}$ is a real random variable with continuous c.d.f. $F$, independent of $\theta$ and the noise in the agents' signals. $\omega_{t}$ can be interpreted as a shock to the fundamentals (i.e., the strength of the regime) and $\delta$ as the volatility of these shocks. Denoting with $\Gamma(\delta)$ the game in which the volatility is $\delta$, the baseline model can be nested as $\delta=0$.

Equilibrium characterization with $\delta>0$. In the presence of shocks, the regime outcome depends, not only on $\theta$, but also on $\omega_{t}$. As a result, we loose the ability to characterize the set of monotone equilibria in terms of a sequence of truncation points for $\theta$. Nevertheless, we can still characterize strategies as sequences of thresholds $\left\{\bar{x}_{t}\right\}_{t=1}^{\infty}$ such that an agent attacks in period $t$ if and only if $x_{t} \leq \bar{x}_{t}$, where $\bar{x}_{t} \in \overline{\mathbb{R}}$.

Consider first the regime outcomes induced by a given $\left\{\bar{x}_{t}\right\}_{t=1}^{\infty}$. The size of the attack in period $t$ is $A_{t}(\theta)=\Phi\left(\sqrt{\alpha_{t}}\left(\bar{x}_{t}-\theta\right)\right)$ and hence the status quo is abandoned if and only if $\omega_{t} \leq$ $\bar{\omega}_{t}\left(\theta ; \bar{x}_{t}, \delta\right) \equiv\left[\Phi\left(\sqrt{\alpha_{t}}\left(\bar{x}_{t}-\theta\right)\right)-\theta\right] / \delta$. It follows that the probability of regime change in period $t$ conditional on $\theta$ and given that agents use monotone strategies with threshold $\bar{x}_{t}$, is

$$
p_{t}\left(\theta ; \bar{x}_{t}, \delta\right)=F\left(\bar{\omega}_{t}\left(\theta ; \bar{x}_{t}, \delta\right)\right) .
$$

Next, for any $t \geq 2$, let $\psi_{t}\left(\theta ; \bar{x}^{t-1}, \delta\right)$ denote the density of the common posterior about $\theta$, when in previous periods agents followed monotone strategies with thresholds $\bar{x}^{t-1}=\left\{\bar{x}_{1}, \ldots, \bar{x}_{t-1}\right\}$. By Bayes' rule,

$\psi_{t}\left(\theta ; \bar{x}^{t-1}, \delta\right)=\frac{\left[1-p_{t-1}\left(\theta ; \bar{x}_{t-1}, \delta\right)\right] \psi_{t-1}\left(\theta ; \bar{x}^{t-2}, \delta\right)}{\int_{-\infty}^{+\infty}\left[1-p_{t-1}\left(\theta^{\prime} ; \bar{x}_{t-1}, \delta\right)\right] \psi_{t-1}\left(\theta^{\prime} ; \bar{x}^{t-2}, \delta\right) d \theta^{\prime}}=\frac{\Pi_{s=1}^{t-1}\left[1-p_{s}\left(\theta ; \bar{x}_{s}, \delta\right)\right] \psi_{1}(\theta)}{\int_{-\infty}^{+\infty} \Pi_{s=1}^{t-1}\left[1-p_{s}\left(\theta^{\prime} ; \bar{x}_{s}, \delta\right)\right] \psi_{1}\left(\theta^{\prime}\right) d \theta^{\prime}}$

where $\psi_{1}(\theta)=\sqrt{\beta} \phi(\sqrt{\beta}(\theta-z))$ is the density of the initial prior. We similarly denote the density of the corresponding private posteriors with $\psi_{t}\left(\theta \mid x ; \bar{x}^{t-1}, \delta\right)$ for $t \geq 2$ and $\psi_{1}(\theta \mid x)$ for $t=1$.

Finally, consider payoffs. For any $t \geq 1, x \in \mathbb{R}$, and $\bar{x}^{t} \in \overline{\mathbb{R}}^{t}$, define $v_{t}\left(x ; \bar{x}^{t}, \delta\right)$ as the net expected payoff from attacking in period $t$ for an agent with sufficient statistic $x$ when all other 
agents attack in period $\tau \leq t$ if and only if their sufficient statistic in $\tau$ is less than or equal to $\bar{x}_{\tau}$ :

$$
v_{t}\left(x ; \bar{x}^{t}, \delta\right)=\int_{-\infty}^{+\infty} p_{t}\left(\theta ; \bar{x}_{t}, \delta\right) \psi_{t}\left(\theta \mid x ; \bar{x}^{t-1}, \delta\right) d \theta-c .
$$

Note that $v_{t}\left(x ; \bar{x}^{t}, \delta\right)$ depends on both the contemporaneous threshold $\bar{x}_{t}$ and the sequence of past thresholds $\bar{x}^{t-1}$; the former determines the probability of regime change conditional on $\theta$, whereas the latter determines the posterior beliefs about $\theta$. Next, for any $t \geq 1$ and $\bar{x}^{t} \in \overline{\mathbb{R}}^{t}$, let

$$
V_{t}\left(\bar{x}^{t} ; \delta\right) \equiv \begin{cases}\lim _{x \rightarrow+\infty} v_{t}\left(x ; \bar{x}^{t}, \delta\right) & \text { if } \bar{x}_{t}=+\infty \\ v_{t}\left(\bar{x}_{t} ; \bar{x}^{t}, \delta\right) & \text { if } \bar{x}_{t} \in \mathbb{R} \\ \lim _{x \rightarrow-\infty} v_{t}\left(x ; \bar{x}^{t}, \delta\right) & \text { if } \bar{x}_{t}=-\infty\end{cases}
$$

$V_{t}$ is the analogue of the function $U$ in the benchmark model: it represents the net payoff from attacking in period $t$ for the marginal agent with threshold $\bar{x}_{t}{ }^{10}$

In Lemma A2 in the Appendix, we prove that, for any $\delta>0, V_{t}\left(\bar{x}^{t} ; \delta\right)$ is continuous in $\bar{x}^{t}$ for any $\bar{x}^{t} \in \overline{\mathbb{R}}^{t-1} \times \mathbb{R}$, which we use to establish the existence, and complete the characterization, of monotone equilibria.

Proposition 3 For any $\delta>0,\left\{a_{t}(\cdot)\right\}_{t=1}^{\infty}$ is a monotone equilibrium for $\Gamma(\delta)$ if and only if there exists a sequence $\left\{\bar{x}_{t}\right\}_{t=1}^{\infty}$ such that:

(i) for all $t, a_{t}(\cdot)=1$ if $x_{t}<\bar{x}_{t}$ and $a_{t}(\cdot)=0$ if $x_{t}>\bar{x}_{t}$;

(ii) for $t=1, \bar{x}_{1} \in \mathbb{R}$ and $V_{1}\left(\bar{x}_{1} ; \delta\right)=0$;

(iii) for any $t \geq 2$, either $\bar{x}_{t}=-\infty$ and $V_{t}\left(\bar{x}^{t} ; \delta\right) \leq 0$, or $\bar{x}_{t} \in \mathbb{R}$ and $V_{t}\left(\bar{x}^{t} ; \delta\right)=0$.

An equilibrium exists for any $\delta>0$.

Convergence to benchmark game. We next consider the properties of the equilibrium set as $\delta \rightarrow 0$. Let $\theta_{t}\left(\bar{x}_{t}\right)$ be the unique solution to $\theta=\Phi\left(\sqrt{\alpha_{t}}\left(\bar{x}_{t}-\theta\right)\right)$, with $\theta_{t}(-\infty)=0$ and $\theta_{t}(+\infty)=1$, and $\theta_{t}\left(\bar{x}^{t}\right)=\max _{\tau \leq t}\left\{\theta_{\tau}\left(\bar{x}_{t}\right)\right\}$. As $\delta \rightarrow 0$, the dependence of the regime outcome on the shock $\omega_{t}$ vanishes: for any $\bar{x}_{t}$ and any $\theta \neq \theta_{t}\left(\bar{x}_{t}\right)$,

$$
p_{t}\left(\theta ; \bar{x}_{t}, \delta\right) \rightarrow p_{t}\left(\theta ; \bar{x}_{t}, 0\right) \equiv \begin{cases}1 & \text { if } \theta \leq \theta_{t}\left(\bar{x}_{t}\right) \\ 0 & \text { if } \theta>\theta_{t}\left(\bar{x}_{t}\right)\end{cases}
$$

It follows that the common posterior in any period $t \geq 2$ converges pointwise to the truncated Normal generated by the knowledge that $\theta>\theta_{t-1}\left(\bar{x}^{t-1}\right)$ : for any $\bar{x}^{t-1}$ and any $\theta$,

$$
\psi_{t}\left(\theta ; \bar{x}^{t-1}, \delta\right) \rightarrow \psi_{t}\left(\theta ; \bar{x}^{t-1}, 0\right) \equiv \begin{cases}0 & \text { if } \theta \leq \theta_{t-1}\left(\bar{x}^{t-1}\right) \\ \frac{\sqrt{\beta} \phi(\sqrt{\beta}(\theta-z))}{1-\Phi\left(\sqrt{\beta}\left(\theta_{t-1}\left(\bar{x}^{t-1}\right)-z\right)\right)} & \text { otherwise }\end{cases}
$$

\footnotetext{
${ }^{10}$ Since the distribution of $x$ given $\theta$ satisfies the MLRP and $p_{t}\left(\theta ; \bar{x}_{t}, \delta\right)$ is decreasing in $\theta$, by standard representation theorems (Milgrom, 1981), $v_{t}\left(x ; \bar{x}^{t}, \delta\right)$ is decreasing in $x$. Since $v_{t}$ is also bounded in $[-c, 1-c]$, it follows that $\lim _{x \rightarrow \pm \infty} v_{t}\left(x ; \bar{x}^{t}, \delta\right)$ exist for any $\bar{x}^{t} \in \overline{\mathbb{R}}^{t}$ and therefore $V_{t}\left(\bar{x}^{t}, \delta\right)$ is well-defined at $\bar{x}_{t}= \pm \infty$.
} 
Similarly, for the private posteriors, $\psi_{t}\left(\theta \mid x ; \bar{x}^{t-1}, \delta\right) \rightarrow \psi_{t}\left(\theta \mid x ; \bar{x}^{t-1}, 0\right)$ for any $x$.

The pointwise convergence of $p_{t}$ and $\psi_{t}$ in turn implies pointwise convergence of the payoff of the marginal agent except when $t \geq 2$ and $\bar{x}_{t}=-\infty$. In particular, for $t=1$ and any $\bar{x}_{1}$, $V_{1}\left(\bar{x}_{1} ; \delta\right) \rightarrow V_{1}\left(\bar{x}_{1} ; 0\right) \equiv U\left(\theta_{1}\left(\bar{x}_{1}\right),-\infty, \alpha_{1}, \beta, z\right)$; and, for any $t \geq 2$, any $\bar{x}^{t-1}$ and any $\bar{x}_{t}>-\infty$,

$$
V_{t}\left(\bar{x}^{t} ; \delta\right) \rightarrow V_{t}\left(\bar{x}^{t} ; 0\right) \equiv U\left(\theta_{t}\left(\bar{x}_{t}\right), \theta_{t-1}\left(\bar{x}^{t-1}\right), \alpha_{t}, \beta, z\right)
$$

To understand why $V_{t}\left(\bar{x}^{t} ; \delta\right)$ need not converge to $V_{t}\left(\bar{x}^{t} ; 0\right)=-c$ when $t \geq 2$ and $\bar{x}_{t}=-\infty$, note that, in the presence of shocks, an agent with sufficiently low $x_{t}$ may attach probability higher than $c$ to regime change in period $t \geq 2$ even if he expects no other agent to attack in that period. When this is the case, a positive measure of agents may attack in every period in the perturbed game, unlike the benchmark model. Nevertheless, the pointwise convergence of $V_{t}$ for any $\bar{x}_{t}>-\infty$ ensures that this dominance region vanishes as $\delta \rightarrow 0$.

More generally, we can prove that "essentially" all equilibria $\left\{x_{t}^{*}\right\}_{t=1}^{\infty}$ in the benchmark game $\Gamma(0)$ can be approximated by equilibria $\left\{\bar{x}_{t}(\delta)\right\}_{t=1}^{\infty}$ in the perturbed game $\Gamma(\delta)$ for $\delta>0$ small enough. ${ }^{11}$ The qualification "essentially" is needed because convergence may fail in the knife-edge case where $V_{t}\left(x^{* t} ; 0\right)$ reaches its maximum at $x_{t}^{*}$ (or equivalently $U\left(\theta_{t}^{*}, \theta_{t-1}^{*}, \alpha_{t}, \beta, z\right.$ ) reaches its maximum at $\theta_{t}^{*}$ ).

Theorem 2 (Shocks in fundamentals) For any $\varepsilon>0$, and any $T \geq 1$, there exists $\delta(\varepsilon, T)>0$ such that, for all $\delta<\delta(\varepsilon, T)$, the following is true:

For any equilibrium $\left\{x_{t}^{*}\right\}_{t=1}^{\infty}$ of $\Gamma(0)$ such that $x_{t}^{*} \notin \arg \max _{x} V_{t}\left(x^{* t-1}, x ; 0\right)$ for all $t \in\{2, \ldots, T\}$, there exists an equilibrium $\left\{\bar{x}_{t}(\delta)\right\}_{t=1}^{\infty}$ of $\Gamma(\delta)$ such that, for all $t \leq T$, either $\left|x_{t}^{*}-\bar{x}_{t}(\delta)\right|<\varepsilon$, or $x_{t}^{*}=-\infty$ and $\bar{x}_{t}(\delta)<-1 / \varepsilon$.

The above establishes convergence of equilibrium play in the first $T$ periods of the infinitehorizon game, for any finite $T$. Since $\delta(\varepsilon, T)$ may converge to 0 as $T \rightarrow \infty$, the result need not hold for an infinite number of periods. Of course, if the game ended for exogenous reasons at $T$, the above would immediately imply convergence in all periods of the finite-horizon game.

The result is illustrated in Figures 3 and 4 for $T=2$. The solid line in Figure 3 represents the p.d.f. of the common posterior in period 2 generated by equilibrium play in period 1 in the game without shocks $(\delta=0)$. This is simply the initial prior truncated at $\theta_{1}^{*}=\theta_{1}\left(x_{1}^{*}\right)$, where $x_{1}^{*}$ is the unique solution to $V_{1}\left(x_{1}^{*} ; 0\right)=0$ (or equivalently where $\theta_{1}^{*}$ is the unique solution to $\left.U\left(\theta_{1}^{*},-\infty, \alpha_{1}, \beta, z\right)=0\right)$. The other two lines represent the equilibrium common posteriors $\psi_{2}\left(\theta ; \bar{x}_{1}(\delta), \delta\right)$ for the game with shocks $(\delta>0)$, where $\bar{x}_{1}(\delta)$ is the unique solution to $V_{1}\left(\bar{x}_{1}(\delta) ; \delta\right)=0$; the dotted line corresponds to a relatively high $\delta$ and the dashed one to a low

\footnotetext{
${ }^{11}$ Clearly, Proposition 3 applies also to the unperturbed game: $\left\{x_{t}^{*}\right\}_{t=1}^{\infty}$ is a monotone equilibrium of $\Gamma(0)$ if and only it satisfies $(i i)-(i i i)$ for $\delta=0$.
} 


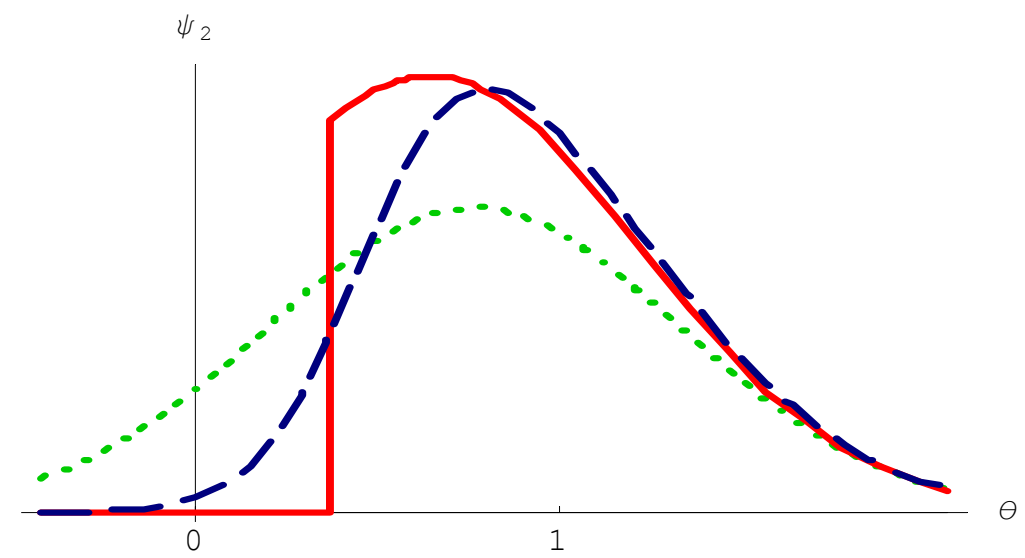

Figure 3: Common posteriors with and without shocks.

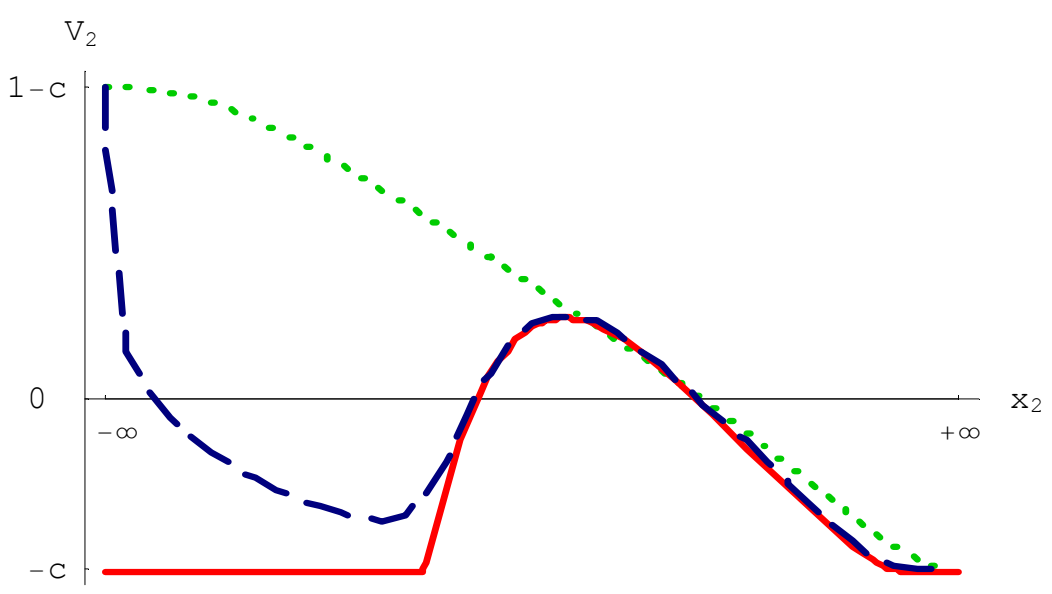

Figure 4: Payoff of the marginal agent with and without shocks.

$\delta$. Since the support of $\omega_{t}$ is the entire real line, the probability of regime change is less than 1 for any $\theta$ and therefore $\psi_{2}$ assigns positive probability to all $\theta$. However, as $\delta$ becomes smaller, $\bar{x}_{1}(\delta)$ converges to $x_{1}^{*}$ and the probability of regime change converges to 1 for $\theta<\theta_{1}^{*}$ and to 0 for $\theta>\theta_{1}^{*}$. By implication, the smooth common posterior of the perturbed game in period 2 converges to the truncated one of the benchmark model.

In Figure 4, the solid line represents the payoff $V_{2}\left(x_{1}^{*}, x_{2} ; 0\right)$ of the marginal agent in period 2 for $\delta=0$, whereas the other two lines represent $V_{2}\left(\bar{x}_{1}(\delta), x_{2} ; \delta\right)$ for $\delta>0 .{ }^{12}$ Note that, for $x_{2}$ small enough, $V_{2}$ is negative when $\delta=0$ but positive when $\delta>0$, which implies that nobody attacking in period 2 is part of an equilibrium in the benchmark model but not in the game with

\footnotetext{
${ }^{12}$ In order to illustrate $V_{2}$ over its entire domain, the figure depicts $V_{2}\left(\bar{x}_{1}(\delta), x_{2} ; \delta\right)$ against $\Phi\left(x_{2}\right)$ rather than $x_{2}$.
} 
shocks. ${ }^{13}$ Moreover, when $\delta$ is high (dotted line), $V_{2}$ is monotonic in $x_{2}$ and therefore has a single intersection with the horizontal line, in which case the equilibrium would be unique if the game ended in period 2. When, instead, $\delta$ is sufficiently small (dashed line), $V_{2}$ is non-monotonic and has three intersections, which correspond to three different equilibria for the two-period game with shocks. Finally, the middle and the highest intersections approximate the two intersections of the solid line, while the lowest intersection is arbitrarily small, thus approximating $x_{2}^{*}=-\infty$. Along with the fact that $\bar{x}_{1}(\delta)$ converges to $x_{1}^{*}$, this implies that any equilibrium of the two-period game without shocks can be approximated by an equilibrium in the perturbed game.

In conclusion, what sustains multiplicity in the dynamic game is the non-monotonicity of the payoff of the marginal agent generated by the knowledge that the regime survived past attacks. That this knowledge resulted in posterior beliefs that assign zero measure to sufficiently low $\theta$ in the benchmark model, is not essential.

\section{Public news and signals about past attacks}

In this section, we consider two extensions of the information structure assumed in the benchmark model.

\subsection{Exogenous public signals}

To capture the effect of public news, we now modify the game as follows. In addition to their private signals, agents observe in each period $t \geq 1$ a public signal $\tilde{z}_{t}=\theta+\varepsilon_{t}$, where $\varepsilon_{t}$ is common noise, normally distributed with zero mean and precision $\eta_{t}^{z}>0$, serially uncorrelated, and independent of $\theta$ and the agent's private noise. These signals may represent, for example, the information generated by news analysis in the media, publication of government statistics, or announcements by policy makers. We also allow for the possibility that the game ends for exogenous reasons at a finite date and denote the horizon of the game with $T$, where $T \in\{2,3, \ldots\}$ or $T=\infty$.

The common posterior about $\theta$ conditional on $\tilde{z}^{t} \equiv\left\{\tilde{z}_{\tau}\right\}_{\tau=1}^{t}$ is Normal with mean $z_{t}$ and precision $\beta_{t}$, where

$$
z_{t}=\frac{\beta_{t-1}}{\beta_{t}} z_{t-1}+\frac{\eta_{t}^{z}}{\beta_{t}} \tilde{z}_{t}, \quad \beta_{t}=\beta_{t-1}+\eta_{t}^{z},
$$

with $\left(z_{0}, \beta_{0}\right)=(z, \beta)$. However, since equilibrium play in past periods now depends on the realizations of past public signals, $z_{t}$ is not a sufficient statistic conditional on $R_{t}=0$. We thus allow agents to condition their actions on the entire sequence $\tilde{z}^{t}$, or equivalently on $z^{t} \equiv\left\{z_{\tau}\right\}_{\tau=1}^{t}$. Apart

\footnotetext{
${ }^{13}$ In the examples in Figures 3 and 4, an agent finds it optimal to attack in period 2 for sufficiently low $x_{2}$, even if he expects no other agent to attack. However, this need not be the case if $\omega_{t}$ had a bounded support and $\delta$ were small enough.
} 
from this modification, the set of monotone equilibria can be constructed in a similar way as in the benchmark model.

Proposition $4\left\{a_{t}(\cdot)\right\}_{t=0}^{T}$ is a monotone equilibrium if and only if there exists a sequence of functions $\left\{x_{t}^{*}, \theta_{t}^{*}\right\}_{t=1}^{T}$, with $x_{t}^{*}: \mathbb{R}^{t} \rightarrow \overline{\mathbb{R}}$ and $\theta_{t}^{*}: \mathbb{R}^{t} \rightarrow(0,1)$, such that:

(i) for all $t, a_{t}(\cdot)=1$ if $x_{t}<x_{t}^{*}\left(z^{t}\right)$ and $a_{t}(\cdot)=0$ if $x_{t}>x_{t}^{*}\left(z^{t}\right)$;

(ii) at $t=1, \theta_{1}^{*}\left(z_{1}\right)$ solves $U\left(\theta_{1}^{*},-\infty, \alpha_{1}, \beta_{1}, z_{1}\right)=0$ and $x_{1}^{*}\left(z_{1}\right)=X\left(\theta_{1}^{*}\left(z_{1}\right), \alpha_{1}\right)$

(iii) at $t \geq 2$, either $\theta_{t}^{*}\left(z^{t}\right)$ solves

$$
U\left(\theta_{t}^{*}, \theta_{t-1}^{*}, \alpha_{t}, \beta_{t}, z_{t}\right)=0
$$

and $x_{t}^{*}\left(z^{t}\right)=X\left(\theta_{t}^{*}\left(z^{t}\right), \alpha_{t}\right)$, or $\theta_{t}^{*}\left(z^{t}\right)=\theta_{t-1}^{*}\left(z^{t-1}\right)$ and $x_{t}^{*}\left(z^{t}\right)=-\infty$.

Like in the benchmark model without public news, there always exist equilibria in which attacks cease after any arbitrary period. However, for any $\left(\theta_{t-1}^{*}, \alpha_{t}, \beta_{t}\right),(9)$ admits a solution if and only if $z_{t} \leq \bar{z}_{t}$, where $\bar{z}_{t}=\bar{z}\left(\theta_{t-1}^{*}, \alpha_{t}, \beta_{t}\right)$ is always finite. Hence, there also exist equilibria in which an attack occurs in period $t$ for sufficiently low realizations of $z_{t}$. The following then extends and reinforces the multiplicity result of Theorem 1 .

Theorem 3 (Public news) In the game with public news, there always exist multiple equilibria, no matter the mean $z$ of the prior, the precisions $\left\{\alpha_{t}, \beta_{t}\right\}_{t=1}^{T}$ of private and public information, and the horizon $T$ of the game.

Consider now how the introduction of public news affects the ability of an "econometrician" to predict the regime outcome and/or the occurrence of an attack in any given period. For any $t$, any $\theta \in(0,1)$, and any $\theta_{t-1}^{*}<\theta,(9)$ admits a solution higher than $\theta$ if and only if $z_{t}$ is low enough, implying that, conditional on $\theta$, the probability that the status quo is abandoned in any given period is strictly between 0 and 1 . It follows that an econometrician who can observe $\theta$ but can not observe $z^{t}$, necessarily faces uncertainty about the event of regime change. On the other hand, if he also knows $z^{t}$, he may be able to perfectly predict the regime outcome in a given period for some combinations of $\theta$ and $z^{t}$, without, however, being able to predict whether an attack will take place. For example, at any $t \geq 2$, if $\theta>\bar{\theta}_{t}\left(z^{t}\right)>\underline{\theta}_{1}\left(z_{1}\right)$, where $\underline{\theta}_{1}\left(z_{1}\right)$ is the lowest solution to $U\left(\theta^{*},-\infty, \alpha_{1}, \beta_{1}, z_{1}\right)=0$ and $\bar{\theta}_{t}\left(z^{t}\right)$ the highest solution to $U\left(\theta^{*}, \underline{\theta}_{1}\left(z_{1}\right), \alpha_{t}, \beta_{t}, z_{t}\right),{ }^{14}$ there is no equilibrium in which the status is abandoned in period $t$, but there are both an equilibrium in which an attack occurs and one in which no attack takes place in that period. The combination of fundamentals and public information may help predict regime outcomes but not the occurrence of attacks, like in the benchmark model.

\footnotetext{
${ }^{14}$ Since $\theta_{t-1}^{*}\left(z^{t-1}\right) \geq \underline{\theta}_{1}\left(z_{1}\right)$ in any equilibrium, $\bar{\theta}_{t}\left(z^{t}\right)$ is an upper bound for $\theta_{t}^{*}\left(z^{t}\right)$.
} 
Finally, note that the threshold $\bar{z}_{t}$, below which (9) admits a solution, decreases with $\theta_{t-1}^{*}$. Hence, an unsuccessful attack, other things equal, causes a discrete increase in the probability the game enters a phase of inaction during which no attack is possible. In this sense, the prediction of the benchmark model that equilibrium dynamics are characterized by the alternation of phases of tranquility and phases of crises, survives the introduction of public news, but the transition from one phase to another is now stochastic, as it depends on the realization of $z^{t}$.

\subsection{Signals about past attacks}

We now introduce an additional source of information by allowing agents to receive private and public signals about the size of past attacks. These signals are, respectively,

$$
\tilde{X}_{i t}=S\left(A_{t-1}, u_{i t}\right) \quad \text { and } \quad \tilde{Z}_{t}=S\left(A_{t-1}, v_{t}\right)
$$

for $t \geq 2$, where $u_{i t}$ is idiosyncratic noise, $v_{t}$ is common noise, and $S:[0,1] \times \mathbb{R} \rightarrow \mathbb{R}$. To preserve Normality of the information structure, we adopt a similar specification as Dasgupta (2002): $S(A, u)=\Phi^{-1}(A)+u, u_{i t} \sim \mathcal{N}\left(0,1 / \gamma_{t}^{x}\right)$, and $v_{t} \sim \mathcal{N}\left(0,1 / \gamma_{t}^{z}\right)$.

Using the property that in any monotone equilibrium $A_{t-1}=\Phi\left(\sqrt{\alpha_{t-1}}\left(x_{t-1}^{*}-\theta\right)\right)$ - where $x_{t-1}^{*}$ may depend on exogenous/endogenous public signals - and following similar steps as in Section 3 , we can again construct sufficient statistics $x_{t}$ and $z_{t}$ with precisions $\alpha_{t}$ and $\beta_{t}$ such that

$$
\theta \mid\left(\tilde{x}^{t}, \tilde{z}^{t}, \tilde{X}^{t}, \tilde{Z}^{t}\right) \sim \mathcal{N}\left(\frac{\alpha_{t}}{\alpha_{t}+\beta_{t}} x_{t}+\frac{\beta_{t}}{\alpha_{t}+\beta_{t}} z_{t}, \frac{1}{\alpha_{t}+\beta_{t}}\right) .
$$

The only difference is that these statistics are now defined by

$$
\begin{gathered}
x_{t}=\frac{\alpha_{t-1}}{\alpha_{t}} x_{t-1}+\frac{\eta_{t}^{x}}{\alpha_{t}} \tilde{x}_{t}+\frac{\mathbf{1}_{t-1} \alpha_{t-1} \gamma_{t}^{x}}{\alpha_{t}}\left\{x_{t-1}^{*}-\frac{1}{\sqrt{\alpha_{t-1}}} \tilde{X}_{t}\right\}, \\
z_{t}=\frac{\beta_{t-1}}{\beta_{t}} z_{t-1}+\frac{\eta_{t}^{z}}{\beta_{t}} \tilde{z}_{t}+\frac{\mathbf{1}_{t-1} \alpha_{t-1} \gamma_{t}^{z}}{\beta_{t}}\left\{x_{t-1}^{*}-\frac{1}{\sqrt{\alpha_{t-1}}} \tilde{Z}_{t}\right\}, \\
\alpha_{t}=\alpha_{t-1}+\eta_{t}^{x}+\mathbf{1}_{t-1} \alpha_{t-1} \gamma_{t}^{x} \quad \text { and } \quad \beta_{t}=\beta_{t-1}+\eta_{t}^{z}+\mathbf{1}_{t-1} \alpha_{t-1} \gamma_{t}^{z},
\end{gathered}
$$

where $\mathbf{1}_{t}$ is an indicator variable that takes value 1 if an attack occurrs at $t\left(A_{t}>0\right)$ and 0 otherwise.

Although the realizations of $\left(x_{t}, z_{t}\right)$ and similarly the dynamics of $\left(\alpha_{t}, \beta_{t}\right)$ are now partly endogenous, the equilibrium characterization is otherwise the same as in the model with only exogenous signals. By implication, the multiplicity results extend to the game with signals about past attacks. Similarly, the structure of equilibrium dynamics remains as in the benchmark model, except for the property that an unsuccessful attack does not necessarily reduce the incentives for further attacks. This is because an unsuccessful attack now also generates new private and public signals, which in some cases may offset the impact of the knowledge that the status quo is still in place.

To see this, consider the case where all signals are private $\left(\gamma_{t}^{x}>0, \eta_{t}^{x} \geq 0, \gamma_{t}^{z}=\eta_{t}^{z}=0\right)$, in which case the only novel effect is that an unsuccessful attack leads to an endogenous increase in 
$\alpha_{t}$. A further attack is then possible only if this increase is large enough, like in the benchmark game. On the other hand, when the endogenous signal is public $\left(\gamma_{t}^{z}>0=\gamma_{t}^{x}\right)$, a new attack becomes possible if this signal is low enough, like in the case with exogenous public news.

We conclude that signals about the size of past attacks may substitute for the exogenous arrival of private and/or public information, but do not otherwise affect the analysis.

\section{Conclusion}

This paper examined how the dynamics of information influences the dynamics of coordination in a global game of regime change. We found that dynamics may sustain multiple equilibria under the same informational assumptions that guarantee uniqueness in the static benchmark examined in the literature.

Although the model is highly stylized and abstracts from all sorts of institutional details and intertemporal payoff linkages, it generates a few distinctive predictions for equilibrium dynamics, such as the possibility that fundamentals predict regime outcomes but not the timing and number of attacks, or the succession of phases of tranquility, during which agents accumulate information and no attacks are possible, and periods of crises, during which attacks may occur but do no necessarily take place. These predictions may help understand the dynamics of crises phenomena such as currency attacks, financial crashes, or political change. Extending the analysis to these applications is a promising line for future research.

\section{Appendix}

Proof of Proposition 1. Solving (1) for $\hat{x}$ gives $\hat{x}=\hat{\theta}+\alpha^{-1 / 2} \Phi^{-1}(\hat{\theta})$. Substituting this into (2) gives a single equation in $\hat{\theta}$ :

$$
U^{s t}(\hat{\theta} ; \alpha, \beta, z)=0
$$

where

$$
U^{s t}(\theta ; \alpha, \beta, z) \equiv 1-\Phi\left(\frac{\sqrt{\alpha}}{\sqrt{\alpha+\beta}}\left[\Phi^{-1}(\theta)+\frac{\beta}{\sqrt{\alpha}}(z-\theta)\right]\right)-c .
$$

Note that $U^{s t}(\theta ; \cdot)$ is continuous and differentiable in $\theta \in(0,1)$, with $\lim _{\theta \rightarrow 0} U^{s t}(\theta)=1-c>0$ and $\lim _{\theta \rightarrow 1} U^{s t}(\theta)=-c<0$. A solution to (10) therefore always exists. Next, note that

$$
\frac{\partial U^{s t}(\theta ; .)}{\partial \theta}=-\frac{\sqrt{\alpha}}{\sqrt{\alpha+\beta}} \phi\left(\frac{\sqrt{\alpha}}{\sqrt{\alpha+\beta}}\left[\Phi^{-1}(\theta)+\frac{\beta}{\sqrt{\alpha}}(z-\theta)\right]\right)\left[\frac{1}{\phi\left(\Phi^{-1}(\theta)\right)}-\frac{\beta}{\sqrt{\alpha}}\right] .
$$

Since $\min _{\theta \in(0,1)}\left[1 / \phi\left(\Phi^{-1}(\theta)\right)\right]=\sqrt{2 \pi}$, the condition $\alpha \geq \beta^{2} /(2 \pi)$ is both necessary and sufficient for $U^{s t}$ to be monotonic in $\theta$, in which case the monotone equilibrium is unique. Finally, for 
the proof that only this equilibrium survives iterated deletion of strictly dominated strategies, see Morris and Shin $(2000,2001)$.

Proof of Lemma 1. We prove the claim by induction. At $t=1$, the result necessarily holds since in any equilibrium, the agents' strategy for $t=1$ is the same as in the static benchmark. Consider next any $t \geq 2$ and suppose that the result holds for any $\tau \leq t-1$. Since $a_{t}(\cdot)$ is non-increasing in $\tilde{x}^{t}, A_{t}(\theta)$ is non-increasing in $\theta$, implying that either $A_{t}(\theta)<\theta$ (and therefore $\left.R_{t+1}=0\right)$ for all $\theta>\theta_{t-1}^{*}$, in which case $\theta_{t}^{*}=\theta_{t-1}^{*}$, or there exists $\theta_{t}^{*}>\theta_{t-1}^{*}$ such that $A_{t}(\theta)<\theta$ if and only if $\theta>\theta_{t}^{*}$. In the former case, $\operatorname{Pr}\left(R_{t+1}=1 \mid x_{t}, R_{t}=0\right)=0$ for all $x_{t}$ and hence $x_{t}^{*}=-\infty$. In the latter,

$$
\operatorname{Pr}\left(R_{t+1}=1 \mid x_{t}, R_{t}=0\right)=\operatorname{Pr}\left(\theta \leq \theta_{t}^{*} \mid x_{t}, \theta>\theta_{t-1}^{*}\right)=1-\frac{\Phi\left(\sqrt{\alpha_{t}+\beta}\left[\frac{\alpha_{t} x_{t}+\beta z}{\alpha_{t}+\beta}-\theta_{t}^{*}\right]\right)}{\Phi\left(\sqrt{\alpha_{t}+\beta}\left[\frac{\alpha_{t} x_{t}+\beta z}{\alpha_{t}+\beta}-\theta_{t-1}^{*}\right]\right)},
$$

which is strictly decreasing in $x_{t}$ and converges to 1 as $x_{t} \rightarrow-\infty$ and to 0 as $x_{t} \rightarrow+\infty$, implying that there exists $x_{t}^{*} \in \mathbb{R}$ such that $\operatorname{Pr}\left(\theta \leq \theta_{t}^{*} \mid x_{t}, \theta>\theta_{t-1}^{*}\right) \geq(\leq) c$ if and only if $x_{t} \leq(\geq) x_{t}^{*}$. In either case, $A_{t}(\theta)<1$ for all $\theta$ and hence $\theta_{t}^{*}<1$, which together with $\theta_{t}^{*} \geq \theta_{1}^{*}>0$, implies that $\theta_{t}^{*} \in(0,1)$ for all $t$, which completes the proof.

Proof of Proposition 2. Necessity follows from the arguments in the main text. For sufficiency, take any sequence $\left\{x_{t}^{*}, \theta_{t}^{*}\right\}_{t=1}^{\infty}$ that satisfies conditions (ii) and (iii); let $\theta_{0}^{*}=-\infty$; suppose all other agents follow strategies as in (i), in which case $R_{t}=0$ if and only if $\theta>\theta_{t-1}^{*}$, for all $t \geq 1$; and consider the best response for an individual agent. If $\theta_{t}^{*}=\theta_{t-1}^{*}$, in which case $t \geq 2, \theta_{t-1}^{*}>0$ and $x_{t}^{*}=-\infty$, then $\operatorname{Pr}\left(R_{t+1}=1 \mid x_{t}, R_{t}=0\right)=\operatorname{Pr}\left(\theta \leq \theta_{t}^{*} \mid x_{t}, \theta>\theta_{t-1}^{*}\right)=0$ for all $x_{t}$ and therefore not attacking is indeed optimal. If instead $\theta_{t}^{*}>\theta_{t-1}^{*}$, in which case $U\left(\theta_{t}^{*}, \theta_{t-1}^{*}, \alpha_{t}, \beta, z\right)=0$ and $x_{t}^{*}=X\left(\theta_{t}^{*}, \alpha_{t}\right)$, then, by the monotonicity of the private posterior and the definitions of $X(\cdot)$ and $U(\cdot), \operatorname{Pr}\left(\theta \leq \theta_{t}^{*} \mid x_{t}, \theta>\theta_{t-1}^{*}\right)-c \geq(\leq) U\left(\theta_{t}^{*}, \theta_{t-1}^{*}, \alpha_{t}, \beta, z\right)$ if and only if $x_{t} \leq(\geq) X\left(\theta_{t}^{*}, \alpha_{t}\right)$ and therefore it is indeed optimal to attack for $x_{t}<x_{t}^{*}$ and not to attack for $x_{t}>x_{t}^{*}$.

Proof of Lemma 2. Part (i) follows directly by inspecting $U$.

For $(i i)$, note that $U\left(\theta^{*}, \theta_{t-1}^{*}, \alpha_{t}, \beta, z\right)<U\left(\theta^{*},-\infty, \alpha_{t}, \beta, z\right)$ for all $\theta^{*}$ (since $\left.\theta_{t-1}^{*}>-\infty\right)$ and that $U\left(\theta^{*},-\infty, \alpha_{t}, \beta, z\right)=U^{s t}\left(\theta^{*}, \alpha_{t}, \beta, z\right)$ is strictly decreasing in $\theta^{*}$ (since $\alpha_{t} \geq \beta^{2} /(2 \pi)$ ). It follows that $U\left(\theta^{*}, \theta_{t-1}^{*}, \alpha_{t}, \beta, z\right)<0$ for all $\theta^{*} \geq \widehat{\theta}_{t}$, which gives the result.

For $(i i i)$, take any $\theta_{t-1}^{*}>\theta_{\infty}$. Note that for all $\theta^{*} \in\left[\theta_{t-1}^{*}, 1\right], \lim _{\alpha \rightarrow \infty} U\left(\theta^{*},-\infty, \alpha, \beta, z\right)=$ $\theta_{\infty}-\theta^{*}<0$. Since $U\left(\theta^{*},-\infty, \alpha, \beta, z\right)$ is continuous in $\theta^{*}$ and $\left[\theta_{t-1}^{*}, 1\right]$ is compact, it follows that there exists $\bar{\alpha}$ such that, for any $\alpha>\bar{\alpha}, U\left(\theta^{*},-\infty, \alpha, \beta, z\right)<0$ for all $\theta^{*} \in\left[\theta_{t-1}^{*}, 1\right]$. Moreover, for all $\alpha, U\left(\theta^{*}, \theta_{t-1}^{*}, \alpha, \beta, z\right)=-c<0$ for $\theta \leq \theta_{t-1}^{*}$ and $U\left(\theta^{*}, \theta_{t-1}^{*}, \alpha, \beta, z\right)<U\left(\theta^{*},-\infty, \alpha, \beta, z\right)$ for $\theta^{*}>\theta_{t-1}^{*}$. It follows that, for any $\alpha>\bar{\alpha}, U\left(\theta^{*}, \theta_{t-1}^{*}, \alpha, \beta, z\right)<0$ for all $\theta^{*}$ and therefore (6) admits no solution. 
For $(i v)$, take any $\theta_{t-1}^{*}<\theta_{\infty}$. Since $\lim _{\alpha \rightarrow \infty} U\left(\theta^{*}, \theta_{t-1}^{*}, \alpha, \beta, z\right)=\theta_{\infty}-\theta^{*}>0$ for any $\theta^{*} \in$ $\left(\theta_{t-1}^{*}, \theta_{\infty}\right)$, there exist $\theta^{\prime} \in\left(\theta_{t-1}^{*}, \theta_{\infty}\right)$ and $\bar{\alpha}$ such that, for any $\alpha>\bar{\alpha}, U\left(\theta^{\prime}, \theta_{t-1}^{*}, \alpha, \beta, z\right)>0$. By the continuity of $U\left(\theta^{*}, \theta_{t-1}^{*}, \alpha, \beta, z\right)$ in $\theta^{*}$ and the fact that $\lim _{\theta^{*} \rightarrow 1} U\left(\theta^{*}, \theta_{t-1}^{*}, \alpha, \beta, z\right)=-c$, it follows then that (6) admits a solution for $\alpha_{t}>\bar{\alpha}$.

Finally, consider $(v)$. Fix $t \geq 2, \theta_{t-2}^{*}, \alpha_{t-1}, \beta$, and $z$ (where we use the convention $\theta_{0}^{*}=$ $-\infty)$ and suppose that $\theta_{t-1}^{*}$ is the highest solution to (6) for period $t-1$, which means that $U\left(\theta^{*}, \theta_{t-2}^{*}, \alpha_{t-1}, \beta, z\right)<0$ for all $\theta^{*}>\theta_{t-1}^{*}$. This, together with the properties that $U\left(\theta^{*}, \theta_{-1}, \alpha, \beta, z\right)$ is non-increasing in $\theta_{-1}$, continuous in $\theta^{*}$, and equal to $-c$ for $\theta^{*} \leq \theta_{-1}$, implies that there exists $\Delta>0$ such that $U\left(\theta^{*}, \theta_{t-1}^{*}, \alpha_{t-1}, \beta, z\right)<-\Delta$ for all $\theta^{*} \in[0,1]$. Furthermore, by continuity of $U$ in $\left(\theta^{*}, \alpha\right)$, there exists $\bar{\alpha}>\alpha_{t-1}$ such that $U\left(\theta^{*}, \cdot, \alpha, \cdot\right)$ is uniformly continuous over $[0,1] \times\left[\alpha_{t-1}, \bar{\alpha}\right]$. This also implies that there exists $\underline{\alpha} \in\left(\alpha_{t-1}, \bar{\alpha}\right)$ such that $U\left(\theta^{*}, \theta_{t-1}^{*}, \alpha, \beta, z\right)<0$ for all $\alpha \in\left[\alpha_{t-1}, \underline{\alpha}\right)$ and all $\theta^{*} \in[0,1]$, which proves that (6) admits no solution in any period $\tau>t$ for which $\alpha_{\tau}<\underline{\alpha}$.

Lemma $\mathbf{A 1}$ There exist thresholds $\underline{z} \leq \bar{z} \leq \overline{\bar{z}}$ such that: $\hat{\theta}_{t} \leq \hat{\theta}_{1}$ for all $t$ if $z \leq \underline{z} ; \hat{\theta}_{1} \leq(\geq) \theta_{\infty}$ if and only $z \geq(\leq) \bar{z}$; and $\hat{\theta}_{t}<\theta_{\infty}$ for all $t$ if and only if $z>\overline{\bar{z}}$. These thresholds satisfy $\underline{z}=\bar{z}=\overline{\bar{z}}$ when $c \leq 1 / 2$ and $\underline{z} \leq \bar{z}<\overline{\bar{z}}$ when $c>1 / 2$.

Proof. For any $\alpha \geq \alpha_{1}\left(\geq \beta^{2} /(2 \pi)\right)$, let $\hat{\theta}=\hat{\theta}(\alpha, \beta, z)$ be the unique solution to $U(\hat{\theta},-\infty, \alpha, \beta, z)=$ 0 (i.e., the static equilibrium threshold) and

$$
\begin{aligned}
& \tilde{z}(\alpha, \beta) \equiv \theta_{\infty}+\frac{\sqrt{\alpha+\beta}-\sqrt{\alpha}}{\beta} \Phi^{-1}\left(\theta_{\infty}\right) \\
& \hat{z}(\alpha, \beta) \equiv \Phi\left(\frac{\sqrt{\alpha}}{\sqrt{\alpha+\beta}} \Phi^{-1}\left(\theta_{\infty}\right)\right)+\frac{1}{\sqrt{\alpha+\beta}} \Phi^{-1}\left(\theta_{\infty}\right) .
\end{aligned}
$$

The threshold $\tilde{z}(\alpha, \beta)$ is defined by $U\left(\theta_{\infty},-\infty, \alpha, \beta, \tilde{z}(\alpha, \beta)\right)=0$ and is such that $\hat{\theta} \geq(\leq) \theta_{\infty}$ if and only if $z \leq(\geq) \tilde{z}$. The threshold $\hat{z}(\alpha, \beta)$, on the other hand, is defined so that $\partial \hat{\theta} / \partial \alpha \geq(\leq) 0$ if and only if $z \geq(\leq) \hat{z}$. To simplify notation, we henceforth suppress the dependence of $\hat{\theta}$ on $(\beta, z)$ and of $\tilde{z}$ and $\hat{z}$ on $\beta$.

First, consider $c=1 / 2$, in which case $\hat{z}(\alpha)=\tilde{z}(\alpha)=1 / 2$ for all $\alpha$. When $z<1 / 2, \hat{\theta}(\alpha)>\theta_{\infty}$ and $\partial \hat{\theta} / \partial \alpha<0$ for all $\alpha \geq \alpha_{1}$ and therefore $\hat{\theta}_{1} \geq \hat{\theta}_{t}>\theta_{\infty}$ for all $t$. When instead $z=1 / 2$, $\hat{\theta}(\alpha)=\theta_{\infty}$ for any $\alpha \geq \alpha_{1}$, and therefore $\hat{\theta}_{1}=\hat{\theta}_{t}=\theta_{\infty}$ for all $t$. Finally, when $z>1 / 2$, for any $\alpha \geq \alpha_{1}, \hat{\theta}(\alpha)<\theta_{\infty}$ and $\partial \hat{\theta} / \partial \alpha>0$, and hence $\hat{\theta}_{1} \leq \hat{\theta}_{t}<\theta_{\infty}$ for all $t$. The result thus holds with $\underline{z}=\bar{z}=\overline{\bar{z}}=1 / 2$.

Next, consider $c<1 / 2$, in which case $\tilde{z}(\alpha)$ and $\hat{z}(\alpha)$ are both decreasing in $\alpha$, satisfy $\hat{z}(\alpha)>$ $\tilde{z}(\alpha)>\theta_{\infty}$ for all $\alpha$, and converge to $\theta_{\infty}$ as $\alpha \rightarrow \infty$. When $z \leq \theta_{\infty}$, then clearly $z<\tilde{z}(\alpha)<\hat{z}(\alpha)$ for all $\alpha$ and therefore $\hat{\theta}(\alpha)$ is always higher than $\theta_{\infty}$ and decreasing in $\alpha$, which implies that $\hat{\theta}_{1} \geq \hat{\theta}_{t}>\theta_{\infty}$ for all $t$. When $z \in\left(\theta_{\infty}, \tilde{z}\left(\alpha_{1}\right)\right)$, there are $\alpha^{\prime \prime}>\alpha^{\prime}>\alpha_{1}$ such that $\tilde{z}\left(\alpha^{\prime}\right)=\hat{z}\left(\alpha^{\prime \prime}\right)=z$. For $\alpha \in\left[\alpha_{1}, \alpha^{\prime}\right), \hat{\theta}(\alpha)$ is higher than $\theta_{\infty}$ and decreases with $\alpha$. As soon as $\alpha \in\left(\alpha^{\prime}, \alpha^{\prime \prime}\right), \hat{\theta}(\alpha)$ becomes lower than $\theta_{\infty}$ and continues to decrease with $\alpha$. Once $\alpha \geq \alpha^{\prime \prime}, \hat{\theta}(\alpha)$ starts increasing with 
$\alpha$, but never exceeds $\theta_{\infty}$. Hence, $\hat{\theta}_{1}>\theta_{\infty}$ and $\hat{\theta}_{1} \geq \hat{\theta}_{t}$ for all $t$. When $z=\tilde{z}\left(\alpha_{1}\right), \hat{\theta}_{1}=\theta_{\infty} \geq \hat{\theta}_{t}$ for all $t$. Finally, when $z>\tilde{z}\left(\alpha_{1}\right), \hat{\theta}(\alpha)<\theta_{\infty}$ for all $\alpha$, and therefore $\hat{\theta}_{t}<\theta_{\infty}$ for all $t$. We conclude that the result holds for $c<1 / 2$ with $\underline{z}=\bar{z}=\overline{\bar{z}}=\tilde{z}\left(\alpha_{1}\right)$.

Finally, consider $c>1 / 2$, in which case $\hat{z}(\alpha)$ and $\tilde{z}(\alpha)$ are both increasing in $\alpha$, satisfy $\hat{z}(\alpha)<\tilde{z}(\alpha)<\theta_{\infty}$, and converge to $\theta_{\infty}$ as $\alpha \rightarrow \infty$. When $z \leq \hat{z}\left(\alpha_{1}\right)$, then clearly $z<\hat{z}(\alpha)<\tilde{z}(\alpha)$ for all $\alpha>\alpha_{1}$ and therefore $\hat{\theta}(\alpha)$ is always higher than $\theta_{\infty}$ and decreasing in $\alpha$, which implies that $\hat{\theta}_{1} \geq \hat{\theta}_{t}>\theta_{\infty}$ for all $t$. When $z \in\left(\hat{z}\left(\alpha_{1}\right), \tilde{z}\left(\alpha_{1}\right)\right)$, there is $\alpha^{\prime}>\alpha_{1}$ such $\hat{z}\left(\alpha^{\prime}\right)=z$. For $\alpha \in\left(\alpha_{1}, \alpha^{\prime}\right), \hat{\theta}(\alpha)$ is higher than $\theta_{\infty}$ and increasing in $\alpha$, whereas for $\alpha>\alpha^{\prime}, \hat{\theta}(\alpha)$ decreases with $\alpha$, converging to $\theta_{\infty}$ from above. It follows that $\max _{t \geq 1} \hat{\theta}_{t} \geq \hat{\theta}_{1}>\theta_{\infty}$. When $z=\tilde{z}\left(\alpha_{1}\right)$, $\max _{t \geq 1} \hat{\theta}_{t} \geq \hat{\theta}_{1}=\theta_{\infty}$. When $z \in\left(\tilde{z}\left(\alpha_{1}\right), \theta_{\infty}\right)$, there are $\alpha^{\prime \prime}>\alpha^{\prime}>\alpha_{1}$ such that $\tilde{z}\left(\alpha^{\prime}\right)=\hat{z}\left(\alpha^{\prime \prime}\right)=z$. For $\alpha \in\left(\alpha_{1}, \alpha^{\prime}\right), \hat{\theta}(\alpha)$ is lower than $\theta_{\infty}$ and increasing in $\alpha$. For $\alpha \in\left(\alpha^{\prime}, \alpha^{\prime \prime}\right), \hat{\theta}(\alpha)$ is higher than $\theta_{\infty}$ and increases with $\alpha$. And for $\alpha>\alpha^{\prime \prime}, \hat{\theta}(\alpha)$ decreases with $\alpha$ and asymptotes to $\theta_{\infty}$ from above. Hence, $\max _{t \geq 1} \hat{\theta}_{t}>\theta_{\infty}>\hat{\theta}_{1}$. Finally, when $z \geq \theta_{\infty}$, then clearly $z>\tilde{z}(\alpha)>\hat{z}(\alpha)$ for all $\alpha$ and therefore $\hat{\theta}(\alpha)$ is always lower than $\theta_{\infty}$, increases with $\alpha$, and asymptotes $\theta_{\infty}$ from below. Hence, $\hat{\theta}_{1} \leq \hat{\theta}_{t}<\theta_{\infty}$ for all $t$. We conclude that the result holds for $c>1 / 2$ with $\underline{z}=\hat{z}\left(\alpha_{1}\right), \bar{z}=\tilde{z}\left(\alpha_{1}\right)$, and $\overline{\bar{z}}=\theta_{\infty}$.

Lemma A2 For any $\delta \geq 0$ and any $t \geq 2, V_{t}\left(\bar{x}^{t} ; \delta\right)$ is continuous in $\bar{x}^{t}$ for any $\bar{x}^{t} \in \overline{\mathbb{R}}^{t-1} \times \mathbb{R}$, and similarly $V_{1}\left(\bar{x}_{1} ; \delta\right)$ is continuous in $\bar{x}_{1}$ for any $\bar{x}_{1} \in \mathbb{R}^{15}$

Proof. Consider first $\delta=0$, in which case $V_{1}(\bar{x} ; 0) \equiv U\left(\theta_{1}(\bar{x}),-\infty, \alpha_{1}, \beta, z\right)$ and $V_{t}\left(\bar{x}^{t} ; 0\right) \equiv$ $U\left(\theta_{t}\left(\bar{x}_{t}\right), \theta_{t-1}\left(\bar{x}^{t-1}\right), \alpha_{t}, \beta, z\right)$ for $t \geq 2$. Note that, for all $t, \theta_{t}\left(\bar{x}_{t}\right)$ is continuous in $\bar{x}_{t} \in \overline{\mathbb{R}}_{\text {and }}$ takes values in $[0,1]$. Since the max operator is continuous, it follows that $\theta_{t}\left(\bar{x}^{t}\right) \equiv \max _{\tau \leq t}\left\{\theta_{\tau}\left(\bar{x}_{\tau}\right)\right\}$ is also continuous in $\bar{x}^{t} \in \overline{\mathbb{R}}^{t}$ and takes values in [0,1]. Furthermore, $U(\theta,-\infty, \alpha, \beta, z)$ is continuous in $\theta \in[0,1]$ and $U\left(\theta, \theta_{-1}, \alpha, \beta, z\right)$ is continuous in $\left(\theta, \theta_{-1}\right) \in[0,1]^{2}$. It follows that, for all $t, V_{t}\left(\bar{x}^{t} ; 0\right)$ is continuous in $\overline{\mathbb{R}}^{t}$.

Consider next $\delta>0$. To simplify notation, we henceforth suppress the dependence of $p_{t}$, $v_{t}$ and $V_{t}$ on $\delta$ whenever there is no risk of confusion. For all $t \geq 1$, the function $p_{t}\left(\theta ; \bar{x}_{t}\right)=$

\footnotetext{
${ }^{15}$ Continuity can be extended in $\overline{\mathbb{R}}^{t}$ as follows. For any function $f: A \rightarrow \overline{\mathbb{R}}$, where $A \subseteq \overline{\mathbb{R}}^{t}$ and $t \geq 1$, we say that $f$ is continuous over $A$ if and only if, for any $x^{t} \in A$ and any $\varepsilon>0$, there exists $\eta>0$ such that, for any $\tilde{x}^{t} \in A$ such that for all $\tau \leq t$ : (a) $\left|\tilde{x}_{\tau}-x_{\tau}\right|<\eta$ if $x_{\tau} \in \mathbb{R}$; (b) $\tilde{x}_{\tau}<-1 / \eta$ if $x_{\tau}=-\infty$; (c) $\tilde{x}_{\tau}>1 / \eta$, if $x_{\tau}=+\infty$, the following is true: (a') if $f\left(x^{t}\right) \in \mathbb{R}$, then $\left|f\left(\tilde{x}^{t}\right)-f\left(x^{t}\right)\right|<\varepsilon$; (b') if $f\left(x^{t}\right)=-\infty$, then $f\left(\tilde{x}^{t}\right)<-1 / \varepsilon$; (c') if $f\left(x^{t}\right)=+\infty$, then $f\left(\tilde{x}^{t}\right)>1 / \varepsilon$.

Note that, if $f: A \rightarrow \overline{\mathbb{R}}, g: B \rightarrow \overline{\mathbb{R}}$, and $q: C \rightarrow \overline{\mathbb{R}}$ are continuous, respectively, in $A, B$ and $C$, where $A \subseteq \overline{\mathbb{R}}^{t}$, $B \subseteq \overline{\mathbb{R}}^{k}$, and $f(A) \times g(B) \subseteq C \subseteq \overline{\mathbb{R}}^{2}$, then the function $w: A \times B \rightarrow \overline{\mathbb{R}}$ defined by $w\left(x^{t}, x^{k}\right)=q\left(f\left(x^{t}\right), g\left(x^{k}\right)\right)$ is continuous in $A \times B$.
} 
$F\left(\left[\Phi\left(\sqrt{\alpha_{t}}\left(\bar{x}_{t}-\theta\right)\right)-\theta\right] / \delta\right)$ is continuous in $\left(\theta, \bar{x}_{t}\right) \in \mathbb{R}^{2}$ and strictly decreasing in $\theta$. For $t \geq 2$

$$
\begin{aligned}
\psi_{t}\left(\theta \mid x ; \bar{x}^{t-1}, \delta\right) & =\frac{\phi\left(\sqrt{\alpha_{t}}(x-\theta)\right) \psi_{t}\left(\theta ; \bar{x}^{t-1}, \delta\right)}{\int_{-\infty}^{+\infty} \phi\left(\sqrt{\alpha_{t}}\left(x-\theta^{\prime}\right)\right) \psi_{t}\left(\theta^{\prime} ; \bar{x}^{t-1}, \delta\right) d \theta^{\prime}} \\
& =\frac{\Pi_{s=1}^{t-1}\left[1-p_{s}\left(\theta ; \bar{x}_{s}\right)\right] \phi\left(\sqrt{\alpha_{t}+\beta}\left(\theta-\frac{\alpha_{t} x+\beta z}{\alpha_{t}+\beta}\right)\right)}{\int_{-\infty}^{+\infty} \Pi_{s=1}^{t-1}\left[1-p_{s}\left(\theta^{\prime} ; \bar{x}_{s}\right)\right] \phi\left(\sqrt{\alpha_{t}+\beta}\left(\theta^{\prime}-\frac{\alpha_{t} x+\beta z}{\alpha_{t}+\beta}\right)\right) d \theta^{\prime}} .
\end{aligned}
$$

The p.d.f. $\psi_{t}-$ and similarly the c.d.f. $\Psi_{t}$ - is thus continuous in $\theta \in \mathbb{R}$ and $\left(x, \bar{x}^{t-1}\right) \in \mathbb{R} \times \overline{\mathbb{R}}^{t-1}$. It follows that $v_{t}\left(x ; \bar{x}^{t-1}, \bar{x}_{t}\right)=\int_{-\infty}^{+\infty} p_{t}\left(\theta ; \bar{x}_{t}\right) d \Psi_{t}\left(\theta \mid x ; \bar{x}^{t-1}, \delta\right)-c$ is continuous in $\left(x, \bar{x}^{t-1}, \bar{x}_{t}\right) \in$ $\mathbb{R} \times \overline{\mathbb{R}}^{t-1} \times \mathbb{R} .^{16}$ It is then immediate that $V_{t}\left(\bar{x}^{t-1}, \bar{x}_{t}\right)=v_{t}\left(\bar{x}_{t} ; \bar{x}^{t-1}, \bar{x}_{t}\right)$ is also continuous in $\left(\bar{x}^{t-1}, \bar{x}_{t}\right) \in \overline{\mathbb{R}}^{t-1} \times \mathbb{R}$. Similarly, for $t=1, v_{1}\left(x ; \bar{x}_{1}\right)$ is continuous in $\left(x, \bar{x}_{1}\right) \in \mathbb{R}^{2}$ and therefore $V_{1}\left(\bar{x}_{1}\right)$ is continuous in $\bar{x}_{1} \in \mathbb{R}$.

Proof of Proposition 3. (To simplify notation, we again suppress the dependence of $p_{t}, v_{t}$ and $V_{t}$ on $\delta$ whenever this does not create confusion.)

Sufficiency. Consider a sequence $\left\{\bar{x}_{t}(\delta)\right\}_{t=1}^{\infty}$ that satisfies conditions $(i i)$ and (iii) in the proposition. The monotonicity of $v_{t}\left(x ; \bar{x}^{t}\right)$ with respect to $x$ (see proof of Lemma A2 above) guarantees that, for any $x \in \mathbb{R}$ and any $\bar{x}^{t} \in \overline{\mathbb{R}}^{t}, v_{t}\left(x ; \bar{x}^{t}\right) \geq(\leq) V_{t}\left(\bar{x}^{t}\right)$ if and only if $x \leq(\geq) \bar{x}_{t}$. It follows that the strategies defined by $(i)-(i i i)$ constitute a monotone equilibrium.

Necessity. Conversely, suppose that $\left\{a_{t}(\cdot)\right\}_{t=1}^{\infty}$ is a monotone equilibrium. Since in any such equilibrium the measure of agents attacking in every period is decreasing in $\theta$, the probability of regime change is also decreasing in $\theta$. Then, by standard representation theorems (Milgrom, 1981), the expected payoff from attacking is decreasing in $x_{t}$, implying that agents must follow cut-off strategies. For $\left\{\bar{x}_{t}\right\}_{t=1}^{\infty}$ to be equilibrium cutoffs, it must be that, for all $t, V_{t}\left(\bar{x}^{t}\right)=0$ if $-\infty<\bar{x}_{t}<+\infty, V_{t}\left(\bar{x}^{t}\right) \leq 0$ if $\bar{x}_{t}=-\infty$, and $V_{t}\left(\bar{x}^{t}\right) \geq 0$ if $\bar{x}_{t}=+\infty$.

We next show that, in any equilibrium, $\bar{x}_{t}<+\infty$ for all $t \geq 1$ and $\bar{x}_{1}>-\infty$. Indeed, if $\bar{x}_{t}=+\infty$, in which case $p_{t}(\theta ;+\infty)=F\left(\frac{1}{\delta}(1-\theta)\right)$, then, for any $t \geq 2,\left(x, \bar{x}^{t-1}\right) \in \mathbb{R} \times \overline{\mathbb{R}}^{t-1}$, and $\theta^{\prime} \in \mathbb{R}$

$$
\begin{aligned}
v_{t}\left(x ; \bar{x}^{t-1},+\infty\right) & =\int_{-\infty}^{+\infty} F\left(\frac{1}{\delta}(1-\theta)\right) \psi_{t}\left(\theta \mid x ; \bar{x}^{t-1}, \delta\right) d \theta-c \\
& =\int_{-\infty}^{\theta^{\prime}} F\left(\frac{1}{\delta}(1-\theta)\right) \psi_{t}\left(\theta \mid x ; \bar{x}^{t-1}, \delta\right) d \theta+\int_{\theta^{\prime}}^{+\infty} F\left(\frac{1}{\delta}(1-\theta)\right) \psi_{t}\left(\theta \mid x ; \bar{x}^{t-1}, \delta\right) d \theta-c \\
& \leq \Psi_{t}\left(\theta^{\prime} \mid x ; \bar{x}^{t-1}, \delta\right)+F\left(\frac{1}{\delta}\left(1-\theta^{\prime}\right)\right)\left[1-\Psi_{t}\left(\theta^{\prime} \mid x ; \bar{x}^{t-1}, \delta\right)\right]-c,
\end{aligned}
$$

\footnotetext{
${ }^{16}$ Note that $v_{t}\left(x ; \bar{x}^{t}\right)$ can also be written as $\int_{0}^{1} F_{p}\left(y ; x, \bar{x}^{t}\right) d y$, where $F_{p}\left(y ; x, \bar{x}^{t}\right)$ is the (probability) measure of $\theta$ for which $p\left(\theta ; \bar{x}_{t}\right)>y$, that is, $F_{p}\left(y ; x, \bar{x}^{t}\right)=0$ if $y=1$ and $F_{p}\left(y ; x, \bar{x}^{t}\right)=\Psi_{t}\left(p^{-1}\left(y ; \bar{x}_{t}\right) \mid x ; \bar{x}^{t-1}, \delta\right)$ if $y \in[0,1)$, where $p^{-1}\left(y ; \bar{x}_{t}\right)$ denotes the inverse of $p\left(\theta ; \bar{x}_{t}\right)$ with respect to $\theta$. The continuities of $p$ and $\Psi_{t}$ then imply that $F_{p}\left(y ; x, \bar{x}^{t}\right)$ is continuous in $\left(x, \bar{x}^{t}\right) \in \mathbb{R} \times \overline{\mathbb{R}}^{t-1} \times \mathbb{R}$, for any $y \in[0,1]$. Since $F_{p}(y ; \cdot)$ is also bounded from above by 1 , from the Dominated Convergence Theorem, it follows that $\int_{0}^{1} F_{p}\left(y ; x, \bar{x}^{t}\right) d y$ is also continuous in $\left(x, \bar{x}^{t}\right)$.
} 
where $\Psi_{t}\left(\theta^{\prime} \mid x ; \bar{x}^{t-1}, \delta\right)=\int_{-\infty}^{\theta^{\prime}} \psi_{t}\left(\theta \mid x ; \bar{x}^{t-1}, \delta\right) d \theta$. Furthermore, since the knowledge that the status quo survived past attacks causes a first-order-stochastic-dominance change in posterior beliefs, ${ }^{17}$ $\Psi_{t}\left(\theta^{\prime} \mid x ; \bar{x}^{t-1}, \delta\right) \leq \Phi\left(\sqrt{\alpha_{t}+\beta}\left(\theta^{\prime}-\frac{\alpha_{t} x+\beta z}{\alpha_{t}+\beta}\right)\right)$. Along with $\lim _{x \rightarrow+\infty} \Phi\left(\sqrt{\alpha_{t}+\beta}\left(\theta^{\prime}-\frac{\alpha_{t} x+\beta z}{\alpha_{t}+\beta}\right)\right)=$ 0 , this implies that $\lim _{x \rightarrow+\infty} v_{t}\left(x ; \bar{x}^{t-1},+\infty\right) \leq F\left(\frac{1}{\delta}\left(1-\theta^{\prime}\right)\right)-c$. Since the latter is true for any $\theta^{\prime} \in \mathbb{R}$, it is also true for $\theta^{\prime} \rightarrow+\infty$, in which case $F\left(\frac{1}{\delta}\left(1-\theta^{\prime}\right)\right) \rightarrow 0$. Together with the fact that $v_{t}$ is bounded from below by $-c$, this implies that $V\left(\bar{x}^{t-1},+\infty\right)=\lim _{x \rightarrow+\infty} v_{t}\left(x ; \bar{x}^{t-1},+\infty\right)=-c<0$ and hence $\bar{x}_{t}=+\infty$ can not be part of any equilibrium. A similar argument rules out $\bar{x}_{1}=+\infty$. Finally, suppose $\bar{x}_{1}=-\infty$. Then, for any $x \in \mathbb{R}$ and any $\theta^{\prime} \in \mathbb{R}$,

$$
v_{1}(x ;-\infty)=\int_{-\infty}^{+\infty} F\left(\frac{1}{\delta}(-\theta)\right) \psi_{1}(\theta \mid x) d \theta-c \geq \Psi_{1}\left(\theta^{\prime} \mid x\right) F\left(\frac{1}{\delta}\left(-\theta^{\prime}\right)\right)-c,
$$

where $\Psi_{1}\left(\theta^{\prime} \mid x\right)=\int_{-\infty}^{\theta^{\prime}} \psi_{1}(\theta \mid x) d \theta$, and therefore $\lim _{x \rightarrow-\infty} v_{1}(x ;-\infty) \geq F\left(\frac{1}{\delta}\left(-\theta^{\prime}\right)\right)-c$. Since this is true also for $\theta^{\prime} \rightarrow-\infty$, and since $v_{1}$ is bounded from above by $1-c$, we have that $V_{1}(-\infty)=$ $\lim _{x \rightarrow-\infty} v_{1}(x ;-\infty)=1-c>0$, implying that $\bar{x}_{1}=-\infty$ can not be part of an equilibrium.

We conclude that $(i)-($ iii $)$ necessarily hold in any monotone equilibrium.

Existence. For any $\delta>0$, the monotonicity of $v_{1}\left(x ; \bar{x}_{1}\right)$ in $\bar{x}_{1}$ along with its continuity in $x$ for any $\bar{x}_{1}$ and the fact that $\lim _{x \rightarrow-\infty} v_{1}(x,-\infty)>0>\lim _{x \rightarrow+\infty} v_{1}(x,+\infty)$, implies that there exist $x^{\prime}, x^{\prime \prime} \in \mathbb{R}$ such that $V_{1}\left(x^{\prime}\right) \geq v_{1}\left(x^{\prime},-\infty\right)>0>v_{1}\left(x^{\prime \prime},+\infty\right) \geq V_{1}\left(x^{\prime \prime}\right)$. The continuity of $V_{1}\left(\bar{x}_{1}\right)$ in $\bar{x}_{1}$ then ensures existence of a solution $\bar{x}_{1}(\delta) \in\left(x^{\prime}, x^{\prime \prime}\right)$ to $V_{1}\left(\bar{x}_{1} ; \delta\right)=0$.

Next, consider $t \geq 2$. For any given $\bar{x}^{t-1}$, a similar argument as above ensures the existence of $x^{\prime \prime} \in \mathbb{R}$ such that $V_{t}\left(\bar{x}^{t-1}, x^{\prime \prime}\right) \leq v_{t}\left(x^{\prime \prime}, \bar{x}^{t-1},+\infty\right)<0$. Moreover, either there also exists $x^{\prime} \in \mathbb{R}$ such that $V_{t}\left(\bar{x}^{t-1}, x^{\prime}\right) \geq 0$, or $V_{t}\left(\bar{x}^{t-1}, \bar{x}_{t}\right)<0$ for all $\bar{x}_{t} \in \mathbb{R}$. In the former case, the continuity of $V_{t}\left(\bar{x}^{t-1}, \bar{x}_{t}\right)$ in $\bar{x}_{t}$ ensures the existence of $\bar{x}_{t} \in\left(x^{\prime}, x^{\prime \prime}\right)$ such that $V_{t}\left(\bar{x}^{t-1}, \bar{x}_{t}\right)=0$. In the latter case, $v_{t}\left(x ; \bar{x}^{t-1},-\infty\right) \leq v_{t}\left(x ; \bar{x}^{t-1}, x\right)=V_{t}\left(\bar{x}^{t-1}, x\right)<0$ for any $x \in \mathbb{R}$ and therefore at $\bar{x}_{t}=-\infty, V_{t}\left(\bar{x}^{t-1},-\infty\right) \equiv \lim _{x \rightarrow-\infty} v_{t}\left(x ; \bar{x}^{t-1},-\infty\right) \leq 0$. We conclude that there exists a sequence $\left\{\bar{x}_{t}(\delta)\right\}_{t=0}^{\infty}$ that satisfies conditions $(i i)$ and (iii) in the proposition.

Proof of Theorem 2. We start by establishing pointwise convergence of $V_{t}$ as $\delta \rightarrow 0$. For any $t \geq 2$ and any $\left(\bar{x}^{t-1}, \bar{x}_{t}\right) \in \overline{\mathbb{R}}^{t-1} \times \mathbb{R}$, by (7) and (8), we have that ${ }^{18}$

$$
\begin{aligned}
\lim _{\delta \rightarrow 0} V_{t}\left(\bar{x}^{t-1}, \bar{x}_{t} ; \delta\right) & =\lim _{\delta \rightarrow 0} \int_{-\infty}^{+\infty} p_{t}\left(\theta ; \bar{x}_{t}, \delta\right) d \Psi_{t}\left(\theta \mid \bar{x}_{t} ; \bar{x}^{t-1}, \delta\right)-c=\Psi_{t}\left(\theta_{t}\left(\bar{x}_{t}\right) \mid \bar{x}_{t} ; \bar{x}^{t-1}, 0\right)-c \\
& =U\left(\theta_{t}\left(\bar{x}_{t}\right) ; \theta_{t-1}\left(\bar{x}^{t-1}\right), \alpha_{t}, \beta, z\right) \equiv V_{t}\left(\bar{x}^{t-1}, \bar{x}_{t} ; 0\right) .
\end{aligned}
$$

\footnotetext{
${ }^{17}$ This can be seen by noting that the ratio of the densities $\psi_{t}\left(\theta \mid x ; \bar{x}^{t-1}, \delta\right) / \sqrt{\alpha_{t}+\beta} \phi\left(\sqrt{\alpha_{t}+\beta}\left(\theta-\frac{\alpha_{t} x+\beta z}{\alpha_{t}+\beta}\right)\right)$ is increasing in $\theta$.

${ }^{18}$ For $\delta>0$, let $F_{p}\left(y ; \bar{x}^{t}, \delta\right)$ be as in footnote 16. Similarly, for $\delta=0, F_{p}\left(y ; \bar{x}^{t}, 0\right)=\Psi_{t}\left(\theta_{t}\left(\bar{x}_{t}\right) \mid \bar{x}_{t} ; \bar{x}^{t-1}, 0\right)$ if $y \in[0,1)$ and $F_{p}\left(y ; \bar{x}^{t}, 0\right)=0$ if $y=1$. Since $F_{p}$ is bounded from above by 1 and, for any $y \in[0,1]$ and any $\bar{x}^{t} \in \overline{\mathbb{R}}^{t-1} \times \mathbb{R}$, $F_{p}\left(y ; \bar{x}^{t}, \delta\right) \rightarrow F_{p}\left(y ; \bar{x}^{t}, 0\right)$ as $\delta \rightarrow 0$, from standard Dominated Convergence Theorems, $\lim _{\delta \rightarrow 0} \int_{0}^{+\infty} F_{p}\left(y ; \bar{x}^{t}, \delta\right) d y=$ $\int_{0}^{+\infty} \lim _{\delta \rightarrow 0} F_{p}\left(y ; \bar{x}^{t}, \delta\right) d y=\int_{0}^{+\infty} F_{p}\left(y ; \bar{x}^{t}, 0\right) d y$, which gives the result.
} 
Similarly, for any $\bar{x}_{1} \in \mathbb{R}$, by $(7)$,

$$
\lim _{\delta \rightarrow 0} V_{1}\left(\bar{x}_{1} ; \delta\right)=\Psi_{1}\left(\theta_{1}\left(\bar{x}_{1}\right) \mid \bar{x}_{1}\right)-c=U\left(\theta_{1}\left(\bar{x}_{1}\right) ;-\infty, \alpha_{1}, \beta, z\right) \equiv V_{1}\left(\bar{x}_{1} ; 0\right) .
$$

We now prove the result by induction. To simplify the notation, for all $t \geq 1$, we henceforth let $U_{t}\left(\bar{x}^{t}\right) \equiv V_{t}\left(\bar{x}^{t} ; 0\right)$ for the unperturbed game $(\delta=0)$ and reserve the use of $V_{t}$ for the perturbed games $(\delta>0)$.

Consider first $T=1$ and fix an arbitrary $\varepsilon>0$. From the strict monotonicity of $U_{1}\left(\bar{x}_{1}\right),{ }^{19}$

$$
U_{1}\left(x_{1}^{*}-\varepsilon\right)>0>U_{1}\left(x_{1}^{*}+\varepsilon\right) .
$$

By the convergence of $V_{1}$ to $U_{1}$ as $\delta \rightarrow 0$, we can find $\delta_{1}(\varepsilon)>0$ such that, for any $\delta<\delta_{1}(\varepsilon)$,

$$
V_{1}\left(x_{1}^{*}-\varepsilon ; \delta\right)>0>V_{1}\left(x_{1}^{*}+\varepsilon ; \delta\right) .
$$

From the continuity of $V_{1}\left(\bar{x}_{1} ; \delta\right)$ in $\bar{x}_{1}$ for any $\delta>0$, it follows that there exists a solution $\bar{x}_{1}(\delta)$ to $V_{1}\left(x_{1} ; \delta\right)=0$ such that $x_{1}^{*}-\varepsilon<\bar{x}_{1}(\delta)<x_{1}^{*}+\varepsilon$. Following the same steps as in the proof of existence in Proposition 3, we can then construct an equilibrium $\left\{\bar{x}_{t}(\delta)\right\}_{t=1}^{\infty}$ for $\Gamma(\delta)$ such that $\left|\bar{x}_{1}(\delta)-x_{1}^{*}\right|<\varepsilon$. This proves the result for $T=1$.

Consider next an arbitrary $T \geq 2$, fix $\varepsilon>0$, and suppose the result holds for $T-1$.

Take first any equilibrium of $\Gamma(0)$ such that $x_{T}^{*}>-\infty$. By the (local) strict monotonicity of $U_{T}$ around $x_{T}^{*}$ implied by the assumption that $x_{T}^{*} \notin \arg \max _{x} U_{T}\left(x^{* T-1}, x\right)$, there exists $\varepsilon_{T}<\varepsilon$ such that either

$$
U_{T}\left(x^{* T-1}, x_{T}^{*}-\varepsilon_{T}\right)>0>U_{T}\left(x^{* T-1}, x_{T}^{*}+\varepsilon_{T}\right),
$$

or $U_{T}\left(x^{* T-1}, x_{T}^{*}-\varepsilon_{T}\right)<0<U_{T}\left(x^{* T-1}, x_{T}^{*}+\varepsilon_{T}\right)$. Without loss of generality, assume the first case - the argument for the other case is identical. From the continuity of $U_{T}\left(x^{T-1}, x_{T}\right)$ in $x^{T-1} \in \overline{\mathbb{R}}^{T-1}$ and the fact that the result holds for $T-1$, there exists some $\varepsilon_{T}^{\prime} \in\left(0, \varepsilon_{T}\right)$ such that, for any $\delta<\delta\left(\varepsilon_{T}^{\prime}, T-1\right)$, there is a sequence $\bar{x}^{T-1}(\delta)$ satisfying the following three conditions: ${ }^{20}$

[C1] for all $t \leq T-1$, either $\bar{x}_{t}(\delta)=-\infty$ and $V_{t}\left(\bar{x}^{\tau}(\delta)\right) \leq 0$, or $\bar{x}_{t} \in \mathbb{R}$ and $V_{\tau}\left(\bar{x}^{\tau}(\delta) ; \delta\right)=0$;

[C2] for all $t \leq T-1,\left|x_{t}^{*}-\bar{x}_{t}(\delta)\right|<\varepsilon_{T}^{\prime}<\varepsilon$ if $x_{t}^{*} \in \mathbb{R}$, and $\bar{x}_{t}(\delta)<-1 / \varepsilon_{T}^{\prime}<-1 / \varepsilon$ if $x_{t}^{*}=-\infty$;

[C3] in period $T, U_{T}\left(\bar{x}^{T-1}(\delta), x_{T}^{*}-\varepsilon_{T}\right)>0>U_{T}\left(\bar{x}^{T-1}(\delta), x_{T}^{*}+\varepsilon_{T}\right)$.

Next, by the convergence of $V_{T}\left(x^{T-1}, x_{T}\right)$ to $U_{T}\left(x^{T-1}, x_{T}\right)$ for any $\left(x^{T-1}, x_{T}\right) \in \overline{\mathbb{R}}^{T-1} \times \mathbb{R}$, there exists $\delta_{T} \in\left(0, \delta\left(\varepsilon_{T}^{\prime}, T-1\right)\right)$ such that, for any $\delta<\delta_{T}$, there is $\bar{x}^{T-1}(\delta)$ that satisfies [C1]-[C2] and such that:

\footnotetext{
${ }^{19}$ This follows from the the monotonicity of $U\left(\theta ;-\infty, \alpha_{1}, \beta, z\right)$ in $\theta$ - which in turn is implied by $\alpha_{1} \geq \beta^{2} / \sqrt{2 \pi}-$ and the monotonicity of $\theta_{1}\left(\bar{x}_{1}\right)$ in $\bar{x}_{1}$.

${ }^{20}$ Continuity of $U$ implies existence of $\varepsilon_{T}^{\prime}$ such that that [C3] holds for any $\bar{x}^{T-1}(\delta)$ that satisfies [C2]; that the result holds for $T-1$ then ensures that, for any $\delta<\delta\left(\varepsilon_{T}^{\prime}, T-1\right)$, there exists $\bar{x}^{T-1}(\delta)$ that satisfies both [C1] and $[\mathrm{C} 2]$.
} 
$\left[\mathrm{C} 3^{\prime}\right]$ in period $T, V_{T}\left(\bar{x}^{T-1}(\delta), x_{T}^{*}-\varepsilon_{T} ; \delta\right)>0>V_{T}\left(\bar{x}^{T-1}(\delta), x_{T}^{*}+\varepsilon_{T} ; \delta\right)$.

But then, by the continuity of $V_{T}\left(x^{T-1}, x_{T}\right)$ in $x_{T}$, for the same $\bar{x}^{T-1}(\delta)$, there exists an $\bar{x}_{T}(\delta) \in \mathbb{R}$, with $\left|x_{T}^{*}-\bar{x}_{T}(\delta)\right|<\varepsilon_{T}<\varepsilon$, that solves $V_{T}\left(\bar{x}^{T-1}(\delta), \bar{x}_{T}(\delta) ; \delta\right)=0$.

Next, take any equilibrium of $\Gamma(0)$ such that $x_{T}^{*}=-\infty$. Recall that, for any $t \geq 2, U_{t}\left(x^{* t-1}, x_{t}\right)=$ $-c<0$ for all $x_{t}<\tilde{x}_{t}$, where $\tilde{x}_{t}>-\infty$ solves $\theta_{t}\left(\tilde{x}_{t}\right)=\theta_{t-1}\left(x^{* t-1}\right) \equiv \max _{\tau \leq t-1} \theta_{\tau}\left(x_{\tau}^{*}\right)$. Pick some $x_{T}^{\prime} \in\left(-\infty, \min \left\{-1 / \varepsilon, \tilde{x}_{T}\right\}\right)$. From the continuity of $U_{T}\left(x^{T-1}, x_{T}\right)$ in $x^{T-1}$ and the fact that the result holds for $T-1$, there exists some $\varepsilon^{\prime} \in(0, \varepsilon)$ such that, for any $\left.\delta<\delta\left(\varepsilon^{\prime}, T-1\right)\right)$, there is a sequence $\bar{x}^{T-1}(\delta)$ which satisfies conditions [C1]-[C2] above (replacing $\varepsilon_{T}^{\prime}$ with $\varepsilon^{\prime}$ ) and such that:

[C4] $U_{T}\left(\bar{x}^{T-1}(\delta), x_{T}^{\prime}\right)<0$.

By the pointwise convergence of $V_{T}$ to $U_{T}$, there also exists a $\left.\delta_{T} \in\left(0, \delta\left(\varepsilon^{\prime}, T-1\right)\right)\right)$ such that, for any $\delta<\delta_{T}$, there is $\bar{x}^{T-1}(\delta)$ that satisfies [C1]-[C2] and such that:

$\left[\mathrm{C}^{\prime}\right] V_{T}\left(\bar{x}^{T-1}(\delta), x_{T}^{\prime} ; \delta\right)<0$.

If, for the same $\bar{x}^{T-1}(\delta)$, there exists an $x_{T}^{\prime \prime} \in\left(-\infty, x_{T}^{\prime}\right)$ such that $V_{T}\left(\bar{x}^{T-1}(\delta), x_{T}^{\prime \prime} ; \delta\right) \geq 0$, then, by the continuity of $V_{T}\left(x^{T-1}, x_{T}\right)$ in $x_{T} \in \mathbb{R}$, there is also an $\bar{x}_{T}(\delta) \in \mathbb{R}$, with $x_{T}^{\prime \prime}<\bar{x}_{T}(\delta)<$ $x_{T}^{\prime}<-1 / \varepsilon$, such that $V_{T}\left(\bar{x}^{T-1}(\delta), \bar{x}_{T}(\delta) ; \delta\right)=0$. If instead $V_{T}\left(\bar{x}^{T-1}(\delta), x_{T} ; \delta\right)<0$ for all $x_{T} \in$ $\left(-\infty, x_{T}^{\prime}\right)$, then $\bar{x}_{T}(\delta)=-\infty$ satisfies $V_{T}\left(\bar{x}^{T-1}(\delta),-\infty\right) \leq 0 .{ }^{21}$

Finally, recall that (6) admits at most two solutions in every $t$ and therefore the set of $x^{T *}$ that can be part of an equilibrium of $\Gamma(0)$ is finite. Hence, there is $\delta(\varepsilon, T) \in(0, \delta(\varepsilon, T-1))$ such that, for any $\delta<\delta(\varepsilon, T)$ and every equilibrium $\left\{x_{t}^{*}\right\}_{t=1}^{\infty}$ of $\Gamma(0)$ for which $x_{t}^{*} \notin \arg \max _{x} U_{t}\left(x^{t-1 *}, x\right)$ for all $t \leq T$, there exists $\bar{x}^{T}(\delta)$ such that, for all $t \leq T$ : if $x_{t}^{*} \in \mathbb{R}$, then $\left|\bar{x}_{t}(\delta)-x_{t}^{*}\right|<\varepsilon$ and $V_{t}\left(\bar{x}^{t}(\delta) ; \delta\right)=0$; and if $x_{t}^{*}=-\infty$, then $\bar{x}_{t}(\delta)<-1 / \varepsilon$ and $V_{t}\left(\bar{x}^{t}(\delta)\right) \leq 0$. Using the same arguments as for the proof of existence in Proposition $3, \bar{x}^{T}(\delta)$ is part of an equilibrium $\left\{\bar{x}_{t}(\delta)\right\}_{t=1}^{\infty}$ for $\Gamma(\delta)$, which completes the proof.

Proof of Proposition 4. Apart from a notational adjustment - namely the dependence of $U$ in period $t$ on $\left(\beta_{t}, z_{t}\right)$ and of $\left(x_{t}^{*}, \theta_{t}^{*}\right)$ on $z^{t}$ - the proof follows exactly the same steps as in the model with only private information, and is thus omitted for brevity.

Proof of Theorem 3. Consider first $t=1$. For any $\left(\alpha_{1}, \beta_{1}, z_{1}\right), U\left(\theta^{*},-\infty, \alpha_{1}, \beta_{1}, z_{1}\right)$ is continuous in $\theta^{*} \in[0,1]$ with $U(0,-\infty, \cdot)=1-c$ and $U(1,-\infty, \cdot)=-c$. Hence a solution $\theta_{1}^{*}\left(z_{1}\right)$ to $U\left(\theta_{1}^{*},-\infty, \alpha_{1}, \beta_{1}, z_{1}\right)=0$ always exists. ${ }^{22}$ Next, consider any $t \geq 2$ and note that, for any $\left(\theta_{t-1}^{*}, \alpha_{t}, \beta_{t}\right)$ and any $\theta^{*} \in\left(\theta_{t-1}^{*}, 1\right), U\left(\theta^{*}, \theta_{t-1}^{*}, \alpha_{t}, \beta_{t}, z_{t}\right)$ is strictly decreasing in $z_{t}$ and $U\left(\theta^{*}, \cdot, z_{t}\right) \rightarrow 1-c>0$ as $z_{t} \rightarrow-\infty$, implying that necessarily $\max _{\theta^{*} \in\left[\theta_{-1}, 1\right]} U\left(\theta^{*}, \cdot, z_{t}\right)>0$ for $z_{t}$ sufficiently low. Furthermore, since $U\left(\theta^{*}, \cdot, z_{t}\right)$ is continuous in $\theta^{*} \in\left[\theta_{t-1}^{*}, 1\right]$ for any $z_{t}$, and since $U\left(\theta^{*}, \theta_{t-1}^{*}, \cdot\right) \rightarrow-c$ monotonically for any $\theta^{*} \in\left[\theta_{t-1}^{*}, 1\right]$ as $z_{t} \rightarrow+\infty$, from stan-

\footnotetext{
${ }^{21}$ This follows from the same argument used in the proof of Proposition 3.

${ }^{22}$ Note that the function $\theta_{1}^{*}(\cdot)$ is unique if and only if $\alpha_{1} \geq \beta_{1}^{2} / 2 \pi$. Hence for $\alpha_{1}<\beta_{1}^{2} / 2 \pi$, the game trivially admits multiple equilibria even if $T=1$.
} 
dard Monotone Convergence Theorems, the function $U\left(\theta^{*}, \theta_{t-1}^{*}, \cdot, z_{t}\right)$ converges uniformly to $-c$ as $z_{t} \rightarrow+\infty$, implying that $\max _{\theta^{*} \in\left[\theta_{-1}, 1\right]} U\left(\theta^{*}, \theta_{t-1}^{*}, \alpha_{t}, \beta_{t}, z_{t}\right)<0$ for $z_{t}$ sufficiently high. The strict monotonicity of $U$ in $z_{t}$ then guarantees that there exists a finite $\bar{z}\left(\theta_{t-1}^{*}, \alpha_{t}, \beta_{t}\right)$ such that $\max _{\theta^{*} \in\left[\theta_{-1}, 1\right]} U\left(\theta^{*}, \theta_{t-1}^{*}, \alpha_{t}, \beta_{t}, z_{t}\right) \geq(\leq) 0$ if and only if $z \leq(\geq) \bar{z}\left(\theta_{t-1}^{*}, \alpha_{t}, \beta_{t}\right)$, which also implies that (9) admits a solution $\theta_{t}^{*}\left(z^{t}\right)>\theta_{t-1}^{*}\left(z^{t-1}\right)$ if and only if $z_{t} \leq \bar{z}\left(\theta_{t-1}^{*}, \alpha_{t}, \beta_{t}\right)$. The following is then an equilibrium: for $t=1, \theta_{1}^{*}\left(z_{1}\right)$ is any solution to $U\left(\theta_{1}^{*},-\infty, \alpha_{1}, \beta_{1}, z_{1}\right)=0$; for all $t \in\{2, \ldots, T\}, \theta_{t}^{*}\left(z^{t}\right)=\max \left(\left\{\theta_{t-1}^{*}\left(z^{t-1}\right)\right\} \cup\left\{\theta^{*}: U\left(\theta^{*}, \theta_{t-1}^{*}\left(z^{t-1}\right), \alpha_{t}, \beta_{t}, z_{t}\right)=0\right\}\right.$. Note that, in this equilibrium, at any $t \geq 2, \theta_{t}^{*}\left(z^{t}\right)>\theta_{t-1}^{*}\left(z^{t-1}\right)$ for all $z_{t} \leq \bar{z}\left(\theta_{t-1}^{*}, \alpha_{t}, \beta_{t}\right)$. Since $\theta_{t}^{*}\left(z^{t}\right)=\theta_{1}^{*}\left(z_{1}\right)$ for

all $z^{t}$ and all $t$ is also an equilibrium, we conclude that the game admits multiple equilibria for any $\left\{\alpha_{t}, \beta_{t}\right\}_{t=1}^{T}$ and any $T \geq 2$.

\section{References}

[1] Angeletos, George-Marios, Christian Hellwig, and Alessandro Pavan (2003) "Coordination and Policy Traps," working paper, MIT/UCLA/Northwestern.

[2] Atkeson, Andrew (2000), "Discussion on Morris and Shin," NBER Macroeconomics Annual.

[3] Carlsson, Hans, and Eric van Damme (1993), "Global Games and Equilibrium Selection," Econometrica 61, 5, 989-1018.

[4] Chamley, Christophe (1999), "Coordinating Regime Switches," Quarterly Journal of Economics 114, 3, 869-905.

[5] Chari, V.V., and Patrick Kehoe (2003), "Hot Money," Journal of Political Economy 111, 1262-1292.

[6] Corsetti, Giancarlo, Bernardo Guimaraes and Nouriel Roubini, (2004), "International Lending of Last Resort and Moral Hazard: A Model of IMF's Catalytic Finance," working paper, University of Rome.

[7] Dasgupta, Amil (2002), "Coordination, Learning and Delay," working paper, London School of Economics.

[8] Edmond, Chris (2003), "Information and the Limits to Autocracy," working paper, UCLA.

[9] Frankel, David, and Ady Pauzner (2000), "Resolving Indeterminacy in Dynamic Settings: The Role of Shocks," Quarterly Journal of Economics 115, 283-304. 
[10] Giannitsarou, Chryssi, and Flavio Toxvaerd (2003), "Recursive Global Games," working paper, Universidade Nova de Lisboa and Hebrew University of Jerusalem.

[11] Goldstein, Itay (2002), "Strategic Complementarities and the Twin Crises," working paper, Duke University.

[12] Goldstein, Itay, and Ady Pauzner (2000), "Demand Deposit Contracts and the Probability of bank Runs," working paper, Duke University and Tel Aviv University.

[13] Goldstein, Itay, and Ady Pauzner (2001), "Contagion of Self-Fulfilling Financial Crises due to Diversification of Investment Portfolios," working paper, Duke University and Tel Aviv University.

[14] Heidhues, Paul, and Nicolas Melissas (2003), "Equilibria in a Dynamic Global Game: The Role of Cohort Effects", working paper, WZB Berlin and University of Leicester.

[15] Levin, Jonathan (2001), "A Note on Global Games with Overlapping Generations," working paper, Stanford University.

[16] Milgrom, Paul (1981), "Good News and Bad News: Representation Theorems and Applications," Bell Journal of Economics 12, 380-391.

[17] Morris, Stephen, and Hyun Song Shin (1998), "Unique Equilibrium in a Model of Self-Fulfilling Currency Attacks," American Economic Review, 88, 3, 587-597.

[18] Morris, Stephen and Hyun Song Shin (1999), "A Theory of the Onset of Currency Attacks," in Agenor, Vines and Weber, eds., Asian Financial Crisis: Causes, Contagion and Consequences, Cambridge, UK: Cambridge University Press.

[19] Morris, Stephen and Hyun Song Shin (2000), "Rethinking Multiple Equilibria in Macroeconomics," NBER Macro Annual.

[20] Morris, Stephen and Hyun Song Shin (2001), "Global Games - Theory and Applications," in M. Dewatripont, L. Hansen, and S. Turnovsky, eds., Advances in Economics and Econometrics (8th World Congress of the Econometric Society), Cambridge, UK: Cambridge University Press.

[21] Morris, Stephen and Hyun Song Shin (2004), "Coordination Risk and the Price of Debt," European Economic Review 48, 133-153.

[22] Rochet, Jean-Charles and Xavier Vives (2004), "Coordination Failures and the Lender of Last Resort: Was Bagehot Right After All?," working paper, University of Toulouse. 